\title{
Asymptotic Approximation of the Nonsteady Micropolar Fluid Flow through a Circular Pipe
}

\author{
Igor Pažanin (iD) and Marko Radulović (i) \\ Department of Mathematics, Faculty of Science, University of Zagreb, Zagreb, Croatia \\ Correspondence should be addressed to Igor Pažanin; pazanin@math.hr
}

Received 27 September 2017; Revised 2 January 2018; Accepted 29 January 2018; Published 11 April 2018

Academic Editor: Michael Vynnycky

Copyright (C) 2018 Igor Pažanin and Marko Radulović. This is an open access article distributed under the Creative Commons Attribution License, which permits unrestricted use, distribution, and reproduction in any medium, provided the original work is properly cited.

\begin{abstract}
We study the nonsteady flow of a micropolar fluid through a thin cylindrical pipe. The asymptotic behaviour of the flow is found via asymptotic analysis with respect to the small parameter $\epsilon$, representing the pipe's thickness. The asymptotic approximation is derived in the form of the explicit formulae for the fluid velocity and microrotation. We also provide the numerical examples in order to visualize the effects of the micropolar nature of the fluid. The illustrations indicate the influence of the micropolarity on the effective flow of the fluid in the whole domain. In particular, those effects are most clearly observed for the velocity approximation near the boundary of the domain.
\end{abstract}

\section{Introduction}

The theory of micropolar fluids was first introduced in $60 \mathrm{~s}$ in the papers by Aero et al. [1] and Eringen [2]. Since then, it has been the case of study and interest of both the engineering and mathematical community. The importance of the model lays in the fact that it takes into account the microstructure of the fluid. The fluid particles with complex shapes of numerous fluids (e.g., liquid crystals, muddy fluids, polymeric suspensions, animal blood, and even water at small scale) exhibit microscopical effects (rotation, shrinking) which cannot be captured by the classical Navier-Stokes model. In order to overcome this limitation, that is, to describe the rotation of the particles, independently of the fluid as a whole, we introduce a new vector field, the angular velocity field of the particles. As a consequence, we obtain a coupled system of equations, with four new viscosities introduced. This non-Newtonian mathematical model obtained in such way describes the behaviour of various real fluids better than the classical Newtonian model. The monograph [3] provides a detailed overview of the mathematical theory underlying the micropolar fluid model.

Due to its practical importance in industrial and engineering applications, micropolar fluid flows have been extensively investigated, in particular in the last decade (see, e.g., [4-9]). In the present paper, we aim to study a timedependent flow of a micropolar fluid through a thin pipe with constant circular cross-section. Traditionally, in order to describe the geometry of such pipes (naturally appearing in numerous applications), a small parameter $\epsilon$ is introduced, representing the pipe's thickness. We start from the assumption that the solution of the governing problem has the so-called micropolar Poiseuille form, and then, following recent results obtained for nonsteady Newtonian flows [1012], we separate the equations by linearity. Employing an asymptotic expansion of the solution in powers of $\epsilon$, we simultaneously solve the obtained equations. Assuming that the external forces depend only on the time variable, we manage to analytically construct the asymptotic solution of the flow in the explicit form. It should be emphasized that deriving the explicit expressions for the fluid velocity and microrotation could be particularly important with regard to numerical simulations.

The paper is organized as follows: in Section 2, we describe the pipe's geometry and the governing micropolar system of equations. Supposing the micropolar Poiseuille form of the solution, we split the problem into the micropolar heat and micropolar inverse problem. In Section 3, we study the micropolar heat problem and construct the asymptotic 
expansion of the solution up to the order $J=2$. In Section 4, we do the same for the micropolar inverse problem, where we additionally have to deal with the pressure. In Section 5, we collect the obtained results and write the asymptotic approximation of the considered problem. Finally, in Section 6, we present some numerical examples and visualize the asymptotic solution.

To conclude, let us provide some more bibliographic remarks on the subject. Steady-state flow of a micropolar fluid through thin domains has been extensively discussed throughout the literature. In particular, this work is a natural continuation of the first author's previous work on the stationary pipe flows; see [13-16]. However, to our knowledge, the results on time-dependent flow have been reported only for simplified settings in which the microrotation is taken to be a scalar function. We refer the reader to [17-20] which merit careful reading. In the present paper we do not impose such constraint leading to a more realistic flow. For that reason, we believe that the obtained result could prove useful in the engineering practice dealing with pipe flows of micropolar fluid. Last but not least, it should be mentioned that theoretical error analysis providing the justification of the asymptotic approximation formally derived in this paper will be brought in the forthcoming paper by the authors of the present paper.

\section{Micropolar Equations}

Let $\epsilon$ be a small positive parameter. We consider a thin straight pipe

$$
\Omega^{\epsilon}=\left\{x \in \mathbb{R}^{3}: x_{1} \in \mathbb{R}, x^{\prime}=\left(x_{2}, x_{3}\right) \in B^{\epsilon}=\epsilon B\right\},
$$

where

$$
B=\left\{x^{\prime} \in \mathbb{R}^{2}:\left|x^{\prime}\right|<R\right\}
$$

is the circular cross-section of the pipe with constant diameter $R>0$. We denote by $\left(x_{1},\left(x_{2}, x_{3}\right)\right) \equiv\left(x_{1}, x^{\prime}\right)$ the Cartesian coordinates, with $x_{1}$ being the direction coinciding with the axis of the pipe (see Figure 1). Let us introduce the following system of equations describing a nonsteady micropolar fluid flow:

$$
\begin{aligned}
& \frac{\partial \mathbf{u}^{\epsilon}}{\partial t}-\left(\nu+v_{r}\right) \Delta \mathbf{u}^{\epsilon}+\left(\mathbf{u}^{\epsilon} \cdot \nabla\right) \mathbf{u}^{\epsilon}+\nabla p^{\epsilon}=2 \nu_{r} \operatorname{rot} \mathbf{w}^{\epsilon} \\
& \quad+\mathbf{f}^{\epsilon} \quad x \in \Omega^{\epsilon}, \\
& \operatorname{div} \mathbf{u}^{\epsilon}=0 \quad x \in \Omega^{\epsilon}, \\
& \frac{\partial \mathbf{w}^{\epsilon}}{\partial t}-\left(c_{a}+c_{d}\right) \Delta \mathbf{w}^{\epsilon}+\left(\mathbf{u}^{\epsilon} \cdot \nabla\right) \mathbf{w}^{\epsilon} \\
& -\left(c_{0}+c_{d}-c_{a}\right) \nabla \operatorname{div} \mathbf{w}^{\epsilon}+4 \mu_{r} \mathbf{w}^{\epsilon}=2 \nu_{r} \operatorname{rot} \mathbf{u}^{\epsilon}+\mathbf{g}^{\epsilon} \\
& \quad x \in \Omega^{\epsilon} .
\end{aligned}
$$

Here $\mathbf{u}^{\epsilon}\left(x_{1}, x^{\prime}, t\right)=\left(u_{1}^{\epsilon}\left(x_{1}, x^{\prime}, t\right), u_{2}^{\epsilon}\left(x_{1}, x^{\prime}, t\right), u_{3}^{\epsilon}\left(x_{1}, x^{\prime}, t\right)\right)$ stands for the velocity field, $p^{\epsilon}\left(x_{1}, x^{\prime}, t\right)$ is the pressure, and $\mathbf{w}^{\epsilon}\left(x^{\prime}, x_{3}, t\right)=\left(w_{1}^{\epsilon}\left(x_{1}, x^{\prime}, t\right), w_{2}^{\epsilon}\left(x_{1}, x^{\prime}, t\right), w_{3}^{\epsilon}\left(x_{1}, x^{\prime}, t\right)\right)$

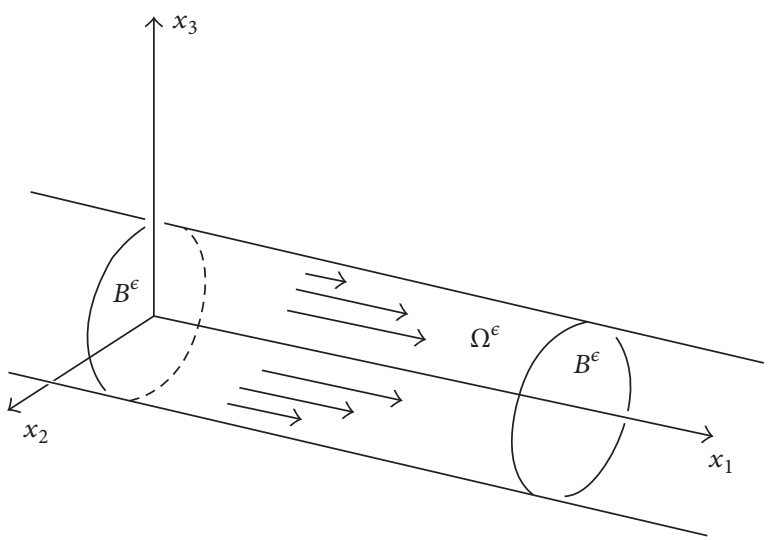

FIgURE 1: The considered flow problem.

is the angular velocity of rotation of the fluid particles (the microrotation field).

To complete the problem, we impose the following boundary and initial conditions:

$$
\begin{aligned}
\mathbf{u}^{\epsilon} & =0, \\
\mathbf{w}^{\epsilon} & =0
\end{aligned}
$$

on $\partial \Omega^{\epsilon}$,

$$
\begin{gathered}
\mathbf{v}^{\epsilon}(x, 0)=\mathbf{a}^{\epsilon}(x), \\
\mathbf{w}^{\epsilon}(x, 0)=\mathbf{b}^{\epsilon}(x, 0),
\end{gathered}
$$

along with the flux condition

$$
\int_{B^{e}} u_{1}^{\epsilon}\left(x_{1}, x^{\prime}, t\right) d x^{\prime}=F(t)
$$

The positive constants are the Newtonian viscosity $v$ and the microrotation viscosity $v_{r}$, while $c_{0}, c_{a}$, and $c_{d}$ represent the coefficients of the angular viscosities. To simplify the notation, we abbreviate $\mu=v+v_{r}, \alpha=c_{a}+c_{d}, \beta=$ $c_{0}+c_{d}-c_{a}, a=2 v_{r}$. The external sources of linear and angular momentum are given by the functions $\mathbf{f}^{\epsilon}=\left(f_{1}^{\epsilon}, f_{2}^{\epsilon}, f_{3}^{\epsilon}\right)$ and $\mathbf{g}^{\epsilon}=\left(g_{1}^{\epsilon}, g_{2}^{\epsilon}, g_{3}^{\epsilon}\right)$, respectively.

As indicated in the Introduction, we assume that the solution of the problem (3) has the micropolar Poiseuille form; namely,

$$
\begin{aligned}
& \mathbf{u}^{\epsilon}(x, t)=\left(v^{\epsilon}\left(x^{\prime}, t\right), 0,0\right), \\
& \mathbf{w}^{\epsilon}(x, t)=\left(0, w_{2}^{\epsilon}\left(x^{\prime}, t\right), w_{3}^{\epsilon}\left(x^{\prime}, t\right)\right), \\
& p^{\epsilon}(x, t)=-q(t) x_{1}+p_{0}(t),
\end{aligned}
$$

where $p_{0}(t)$ is an arbitrary function of $t$. We also assume that $\mathbf{a}^{\epsilon}=\left(a_{1}^{\epsilon}, 0,0\right), \mathbf{b}^{\epsilon}=\left(0, b_{2}^{\epsilon}, b_{3}^{\epsilon}\right), \mathbf{f}^{\epsilon}=\left(f_{1}^{\epsilon}, 0,0\right), \mathbf{g}^{\epsilon}=$ $\left(0, g_{2}^{\epsilon}, g_{3}^{\epsilon}\right)$, with $a_{1}^{\epsilon}=a^{\epsilon}\left(x^{\prime}\right), b_{2}^{\epsilon}=b_{2}^{\epsilon}\left(x^{\prime}\right), b_{3}^{\epsilon}=b_{3}^{\epsilon}\left(x^{\prime}\right)$, $f_{1}^{\epsilon}=f^{\epsilon}\left(x^{\prime}, t\right), g_{2}^{\epsilon}\left(x^{\prime}, t\right)$, and $g_{3}^{\epsilon}\left(x^{\prime}, t\right)$ being independent of the longitudinal variable $x_{1} \in \mathbb{R}$. 
Substituting the micropolar Poiseuille solution (6) into system (3), we obtain the following initial boundary value problem for $x^{\prime} \in B^{\epsilon}$ :

$$
\begin{aligned}
& \frac{\partial p^{\epsilon}}{\partial x_{2}}=0, \\
& \frac{\partial p^{\epsilon}}{\partial x_{3}}=0, \\
& \frac{\partial v^{\epsilon}}{\partial t}-\mu \Delta_{x^{\prime}} \nu^{\epsilon}-q(t)=a\left(\frac{\partial w_{3}^{\epsilon}}{\partial x_{2}}-\frac{\partial w_{2}^{\epsilon}}{\partial x_{3}}\right)+f^{\epsilon}, \\
& \frac{\partial w_{2}^{\epsilon}}{\partial t}-\alpha \Delta_{x^{\prime}} w_{2}^{\epsilon}-\beta \frac{\partial}{\partial x_{2}}\left(\frac{\partial w_{2}^{\epsilon}}{\partial x_{2}}+\frac{\partial w_{3}^{\epsilon}}{\partial x_{3}}\right)+2 a w_{2}^{\epsilon} \\
& \quad=a \frac{\partial v^{\epsilon}}{\partial x_{3}}+g_{2}^{\epsilon}, \\
& \frac{\partial w_{3}^{\epsilon}}{\partial t}-\alpha \Delta_{x^{\prime}} w_{3}^{\epsilon}-\beta \frac{\partial}{\partial x_{3}}\left(\frac{\partial w_{2}^{\epsilon}}{\partial x_{2}}+\frac{\partial w_{3}^{\epsilon}}{\partial x_{3}}\right)+2 a w_{3}^{\epsilon} \\
& \quad=-a \frac{\partial v^{\epsilon}}{\partial x_{2}}+g_{3}^{\epsilon},
\end{aligned}
$$

where $\Delta_{x^{\prime}} v=\partial^{2} v / \partial x_{2}^{2}+\partial^{2} v / \partial x_{3}^{2}$. The corresponding boundary conditions read

$$
\begin{aligned}
\left.v^{\epsilon}\right|_{\partial B^{e}} & =0, \\
\left.w_{2}^{\epsilon}\right|_{\partial B^{\epsilon}} & =0, \\
\left.w_{3}^{\epsilon}\right|_{\partial B^{e}} & =0, \\
v^{\epsilon}\left(x^{\prime}, 0\right) & =a^{\epsilon}\left(x^{\prime}\right), \\
w_{1}^{\epsilon}\left(x^{\prime}, 0\right) & =b_{2}^{\epsilon}\left(x^{\prime}\right), \\
w_{2}^{\epsilon}\left(x^{\prime}, 0\right) & =b_{3}^{\epsilon}\left(x^{\prime}\right),
\end{aligned}
$$

with given flux rate:

$$
\int_{B^{\epsilon}} v^{\epsilon}\left(x^{\prime}, t\right) d x^{\prime}=F(t)
$$

Following the idea from [10-12], we represent the solution $\left(v^{\epsilon}\left(x^{\prime}, t\right), \widehat{\mathbf{w}}^{\epsilon}\left(x^{\prime}, t\right)\right)=\left(v^{\epsilon}\left(x^{\prime}, t\right), w_{2}^{\epsilon}\left(x^{\prime}, t\right), w_{3}^{\epsilon}\left(x^{\prime}, t\right)\right)$ as

$$
\begin{gathered}
v^{\epsilon}\left(x^{\prime}, t\right)=V_{1}^{\epsilon}\left(x^{\prime}, t\right)+V_{2}^{\epsilon}\left(x^{\prime}, t\right), \\
\widehat{\mathbf{w}}^{\epsilon}\left(x^{\prime}, t\right)=\mathbf{W}_{a}^{\epsilon}\left(x^{\prime}, t\right)+\mathbf{W}_{b}^{\epsilon}\left(x^{\prime}, t\right) .
\end{gathered}
$$

Here $\left(V_{1}^{\epsilon}\left(x^{\prime}, t\right), \mathbf{W}_{a}^{\epsilon}\left(x^{\prime}, t\right)\right)=\left(V_{1}^{\epsilon}\left(x^{\prime}, t\right), W_{2,1}^{\epsilon}\left(x^{\prime}, t\right), W_{3,1}^{\epsilon}\left(x^{\prime}, t\right)\right)$ is the solution of the initial boundary value problem for the micropolar heat equation:

$$
\begin{aligned}
& \frac{\partial V_{1}^{\epsilon}}{\partial t}-\mu \Delta_{x^{\prime}} V_{1}^{\epsilon}=a\left(\frac{\partial W_{3,1}^{\epsilon}}{\partial x_{2}}-\frac{\partial W_{2,1}^{\epsilon}}{\partial x_{3}}\right)+f^{\epsilon}, \\
& \frac{\partial W_{2,1}^{\epsilon}}{\partial t}-\alpha \Delta_{x^{\prime}} W_{2,1}^{\epsilon}-\beta \frac{\partial}{\partial x_{2}}\left(\frac{\partial W_{2,1}^{\epsilon}}{\partial x_{2}}+\frac{\partial W_{3,1}^{\epsilon}}{\partial x_{3}}\right) \\
& \quad+2 a W_{2,1}^{\epsilon}=a \frac{\partial V_{1}^{\epsilon}}{\partial x_{3}}+g_{2}^{\epsilon}, \\
& \frac{\partial W_{3,1}^{\epsilon}}{\partial t}-\alpha \Delta_{x^{\prime}} W_{3,1}^{\epsilon}-\beta \frac{\partial}{\partial x_{3}}\left(\frac{\partial W_{2,1}^{\epsilon}}{\partial x_{2}}+\frac{\partial W_{3,1}^{\epsilon}}{\partial x_{3}}\right) \\
& \quad+2 a W_{3,1}^{\epsilon}=-a \frac{\partial V_{1}^{\epsilon}}{\partial x_{2}}+g_{3}^{\epsilon}, \\
& \left.V_{1}^{\epsilon}\right|_{\partial B^{\epsilon}}=0, \\
& \left.W_{2,1}^{\epsilon}\right|_{\partial B^{\epsilon}}=0, \\
& \left.W_{3,1}^{\epsilon}\right|_{\partial B^{\epsilon}}=0, \\
& V_{1}^{\epsilon}\left(x^{\prime}, 0\right)=a^{\epsilon}\left(x^{\prime}\right), \\
& W_{2,1}^{\epsilon}\left(x^{\prime}, 0\right)=b_{2}^{\epsilon}\left(x^{\prime}\right), \\
& W_{3,1}^{\epsilon}\left(x^{\prime}, 0\right)=b_{3}^{\epsilon}\left(x^{\prime}\right) .
\end{aligned}
$$

On the other hand, $\left(V_{2}^{\epsilon}\left(x^{\prime}, t\right), \mathbf{W}_{b}^{\epsilon}\left(x^{\prime}, t\right), q(t)\right)=\left(V_{2}^{\epsilon}\left(x^{\prime}, t\right)\right.$, $\left.W_{2,2}^{\epsilon}\left(x^{\prime}, t\right), W_{3,2}^{\epsilon}\left(x^{\prime}, t\right), q(t)\right)$ denotes the solution of the micropolar inverse problem endowed with the homogeneous initial condition and the given flux rate on the cross-section $B^{\epsilon}$ :

$$
\begin{aligned}
& \frac{\partial V_{2}^{\epsilon}}{\partial t}-\mu \Delta_{x^{\prime}} V_{2}^{\epsilon}=a\left(\frac{\partial W_{3,2}^{\epsilon}}{\partial x_{2}}-\frac{\partial W_{2,2}^{\epsilon}}{\partial x_{3}}\right)+q(t), \\
& \frac{\partial W_{2,2}^{\epsilon}}{\partial t}-\alpha \Delta_{x^{\prime}} W_{2,2}^{\epsilon}-\beta \frac{\partial}{\partial x_{2}}\left(\frac{\partial W_{2,2}^{\epsilon}}{\partial x_{2}}+\frac{\partial W_{3,2}^{\epsilon}}{\partial x_{3}}\right) \\
& \quad+2 a W_{2,2}^{\epsilon}=a \frac{\partial V_{2}^{\epsilon}}{\partial x_{3}}, \\
& \frac{\partial W_{3,2}^{\epsilon}}{\partial t}-\alpha \Delta_{x^{\prime}} W_{3,2}^{\epsilon}-\beta \frac{\partial}{\partial x_{3}}\left(\frac{\partial W_{2,2}^{\epsilon}}{\partial x_{2}}+\frac{\partial W_{3,2}^{\epsilon}}{\partial x_{3}}\right) \\
& \quad+2 a W_{3,2}^{\epsilon}=-a \frac{\partial V_{2}^{\epsilon}}{\partial x_{2}}, \\
& \left.V_{2}^{\epsilon}\right|_{\partial B^{\epsilon}}=0, \\
& \left.W_{2,2}^{\epsilon}\right|_{\partial B^{e}}=0, \\
& \left.W_{3,2}^{\epsilon}\right|_{\partial B^{\epsilon}}=0, \\
& V_{2}^{\epsilon}\left(x^{\prime}, 0\right)=0,
\end{aligned}
$$




$$
\begin{aligned}
& W_{2,2}^{\epsilon}\left(x^{\prime}, 0\right)=0, \\
& W_{3,2}^{\epsilon}\left(x^{\prime}, 0\right)=0, \\
& \int_{B^{\epsilon}} V_{2}^{\epsilon}\left(x^{\prime}, t\right) d x^{\prime}=H(t),
\end{aligned}
$$

where

$$
H(t)=F(t)-\int_{B^{\epsilon}} V_{1}^{\epsilon}\left(x^{\prime}, t\right) d x^{\prime}
$$

From (9) we deduce

$$
H(0)=0 .
$$

Taking into account the problem under consideration, it is plausible to consider the following scaling with respect to small parameter $\epsilon$ :

$$
\begin{aligned}
a^{\epsilon}, b_{2}^{\epsilon}, b_{3}^{\epsilon} & \sim 1, \\
f^{\epsilon} & \sim \epsilon^{-2}, \\
g_{2}^{\epsilon} & \sim \epsilon^{-2}, \\
g_{3}^{\epsilon} & \sim \epsilon^{-2}, \\
F & \sim \epsilon^{2} .
\end{aligned}
$$

In view of that, we expand

$$
\begin{aligned}
\tilde{a}\left(y^{\prime}\right) & =\widetilde{a}^{0}\left(y^{\prime}\right)+\epsilon \widetilde{a}^{1}\left(y^{\prime}\right)+\epsilon^{2} \widetilde{a}^{2}\left(y^{\prime}\right), \\
\tilde{b}_{2}\left(y^{\prime}\right) & =\widetilde{b}_{2}^{0}\left(y^{\prime}\right)+\epsilon \widetilde{b}_{2}^{1}\left(y^{\prime}\right)+\epsilon^{2} \widetilde{b}_{2}^{2}\left(y^{\prime}\right), \\
\widetilde{b}_{3}\left(y^{\prime}\right) & =\widetilde{b}_{3}^{0}\left(y^{\prime}\right)+\epsilon \widetilde{b}_{3}^{1}+\epsilon^{2} \widetilde{b}_{3}^{2}\left(y^{\prime}\right), \\
\widetilde{f}\left(y^{\prime}, t\right) & =\frac{1}{\epsilon^{2}} \widetilde{f}^{0}\left(y^{\prime}, t\right)+\frac{1}{\epsilon} \widetilde{f}^{1}\left(y^{\prime}, t\right)+\widetilde{f}^{2}\left(y^{\prime}, t\right), \\
\widetilde{g}_{2}\left(y^{\prime}, t\right) & =\frac{1}{\epsilon^{2}} \widetilde{g}_{2}^{0}\left(y^{\prime}, t\right)+\frac{1}{\epsilon} \widetilde{g}_{2}^{1}\left(y^{\prime}, t\right)+\widetilde{g}_{2}^{2}\left(y^{\prime}, t\right), \\
\widetilde{g}_{3}\left(y^{\prime}, t\right) & =\frac{1}{\epsilon^{2}} \widetilde{g}_{3}^{0}\left(y^{\prime}, t\right)+\frac{1}{\epsilon} \widetilde{g}_{3}^{1}\left(y^{\prime}, t\right)+\widetilde{g}_{3}^{2}\left(y^{\prime}, t\right), \\
F(t) & =\epsilon^{2} F^{0}(t)+\epsilon^{3} F^{1}(t)+\epsilon^{4} F^{2}(t),
\end{aligned}
$$

and consider problem (7)-(9) with

$$
\begin{aligned}
& a^{\epsilon}(x)=\tilde{a}\left(\frac{x^{\prime}}{\epsilon}\right), \\
& b_{2}^{\epsilon}(x)=\tilde{b}_{2}\left(\frac{x^{\prime}}{\epsilon}\right), \\
& b_{3}^{\epsilon}(x)=\tilde{b}_{3}\left(\frac{x^{\prime}}{\epsilon}\right),
\end{aligned}
$$

$$
\begin{aligned}
& f^{\epsilon}(x, t)=\tilde{f}\left(\frac{x^{\prime}}{\epsilon}, t\right), \\
& g_{2}^{\epsilon}(x, t)=\widetilde{g}_{2}\left(\frac{x^{\prime}}{\epsilon}, t\right), \\
& g_{3}^{\epsilon}(x, t)=\widetilde{g}_{3}\left(\frac{x^{\prime}}{\epsilon}, t\right)
\end{aligned}
$$

and $F(t)$ defined by $(16 \mathrm{~g})$.

\section{Micropolar Heat Problem}

To perform the asymptotic analysis, we first need to rescale the domain, that is, to write the problem on $B$ instead of $B^{\epsilon}$. We introduce the change of variables $y^{\prime}=x^{\prime} / \epsilon$ and obtain the following system of equations deduced from (11):

$$
\begin{aligned}
& \frac{\partial \widetilde{V}_{1}\left(y^{\prime}, t\right)}{\partial t}-\frac{\mu}{\epsilon^{2}} \Delta_{y^{\prime}} \widetilde{V}_{1}\left(y^{\prime}, t\right) \\
& =\frac{a}{\epsilon}\left(\frac{\partial \widetilde{W}_{3,1}\left(y^{\prime}, t\right)}{\partial y_{2}}-\frac{\partial \widetilde{W}_{2,1}\left(y^{\prime}, t\right)}{\partial y_{3}}\right)+\widetilde{f}\left(y^{\prime}, t\right), \\
& \frac{\partial \widetilde{W}_{2,1}\left(y^{\prime}, t\right)}{\partial t}-\frac{\alpha}{\epsilon^{2}} \Delta_{y^{\prime}} \widetilde{W}_{2,1}\left(y^{\prime}, t\right) \\
& -\frac{\beta}{\epsilon^{2}} \frac{\partial}{\partial y_{2}}\left(\frac{\partial \widetilde{W}_{2,1}\left(y^{\prime}, t\right)}{\partial y_{2}}+\frac{\partial \widetilde{W}_{3,1}\left(y^{\prime}, t\right)}{\partial y_{3}}\right) \\
& +2 a \widetilde{W}_{2,1}\left(y^{\prime}, t\right)=\frac{a}{\epsilon} \frac{\partial \widetilde{V}_{1}\left(y^{\prime}, t\right)}{\partial y_{3}}+\widetilde{g}_{2}\left(y^{\prime}, t\right), \\
& \frac{\partial \widetilde{W}_{3,1}\left(y^{\prime}, t\right)}{\partial t}-\frac{\alpha}{\epsilon^{2}} \Delta_{y^{\prime}} \widetilde{W}_{3,1}\left(y^{\prime}, t\right) \\
& -\frac{\beta}{\epsilon^{2}} \frac{\partial}{\partial y_{3}}\left(\frac{\partial \widetilde{W}_{2,1}\left(y^{\prime}, t\right)}{\partial y_{2}}+\frac{\partial \widetilde{W}_{3,1}\left(y^{\prime}, t\right)}{\partial y_{3}}\right) \\
& +2 a \widetilde{W}_{3,1}\left(y^{\prime}, t\right)=-\frac{a}{\epsilon} \frac{\partial \widetilde{V}_{1}\left(y^{\prime}, t\right)}{\partial y_{2}}+\widetilde{g}_{3}\left(y^{\prime}, t\right),
\end{aligned}
$$

where $\widetilde{V}_{1}\left(y^{\prime}, t\right)=V_{1}^{\epsilon}\left(\epsilon y^{\prime}, t\right), \widetilde{W}_{2,1}\left(y^{\prime}, t\right)=W_{2,1}^{\epsilon}\left(\epsilon y^{\prime}, t\right)$, $\widetilde{W}_{3,1}\left(y^{\prime}, t\right)=W_{3,1}^{\epsilon}\left(\epsilon y^{\prime}, t\right)$. The boundary and initial conditions are the following:

$$
\left.\widetilde{V}_{1}\left(y^{\prime}, t\right)\right|_{\partial B}=0,
$$

$$
\begin{aligned}
\left.\widetilde{W}_{2,1}\left(y^{\prime}, t\right)\right|_{\partial B} & =0, \\
\left.\widetilde{W}_{3,1}\left(y^{\prime}, t\right)\right|_{\partial B} & =0, \\
\widetilde{V}_{1}\left(y^{\prime}, 0\right) & =\widetilde{a}\left(y^{\prime}\right), \\
\widetilde{W}_{2,1}\left(y^{\prime}, 0\right) & =\widetilde{b}_{2}\left(y^{\prime}\right), \\
\widetilde{W}_{3,1}\left(y^{\prime}, 0\right) & =\widetilde{b}_{3}\left(y^{\prime}\right) .
\end{aligned}
$$


We rewrite problem (18) as

$$
\begin{aligned}
- & \mu \Delta_{y^{\prime}} \widetilde{V}_{1}\left(y^{\prime}, t\right) \\
& =a \epsilon\left(\frac{\partial \widetilde{W}_{3,1}\left(y^{\prime}, t\right)}{\partial y_{2}}-\frac{\partial \widetilde{W}_{2,1}\left(y^{\prime}, t\right)}{\partial y_{3}}\right) \\
& +\epsilon^{2} \widetilde{f}\left(y^{\prime}, t\right)-\epsilon^{2} \frac{\partial \widetilde{V}_{1}\left(y^{\prime}, t\right)}{\partial t}, \\
- & \alpha \Delta y^{\prime} \widetilde{W}_{2,1}\left(y^{\prime}, t\right) \\
& -\beta \frac{\partial}{\partial y_{2}}\left(\frac{\partial \widetilde{W}_{2,1}\left(y^{\prime}, t\right)}{\partial y_{2}}+\frac{\partial \widetilde{W}_{3,1}\left(y^{\prime}, t\right)}{\partial y_{3}}\right) \\
& =a \epsilon \frac{\partial \widetilde{V}_{1}\left(y^{\prime}, t\right)}{\partial y_{3}}+\epsilon^{2} \widetilde{g}_{2}\left(y^{\prime}, t\right)-\epsilon^{2} \frac{\partial \widetilde{W}_{2,1}\left(y^{\prime}, t\right)}{\partial t} \\
& -2 a \epsilon^{2} \widetilde{W}_{3,1}\left(y^{\prime}, t\right) . \\
- & -2 a \epsilon^{2} \widetilde{W}_{2,1}\left(y^{\prime}, t\right), \\
- & \alpha \Delta y_{y^{\prime}} \widetilde{W}_{3,1}\left(y^{\prime}, t\right) \\
& \left.\partial \frac{\partial \widetilde{V}_{1}\left(y^{\prime}, t\right)}{\partial y_{2}}+\epsilon^{2} \widetilde{g}_{3}\left(y^{\prime}, t\right)-\epsilon^{2} \frac{\partial \widetilde{W}_{3,1}\left(y^{\prime}, t\right)}{\partial t}+\frac{\partial \widetilde{W}_{3,1}\left(y^{\prime}, t\right)}{\partial y_{3}}\right)
\end{aligned}
$$

Now, we construct the formal asymptotic expansion of the solution $\left(\widetilde{V}_{1}^{\mathrm{apr}}\left(y^{\prime}, t\right), \widetilde{W}_{2,1}^{\mathrm{apr}}\left(y^{\prime}, t\right), \widetilde{W}_{3,1}^{\mathrm{apr}}\left(y^{\prime}, t\right)\right)$ in powers of small parameter $\epsilon$ up to $\epsilon^{2}$ in the following way:

$$
\begin{aligned}
\widetilde{V}_{1}^{\mathrm{apr}}\left(y^{\prime}, t\right)= & \widetilde{V}_{1}^{0}\left(y^{\prime}, t\right)+\epsilon \widetilde{V}_{1}^{1}\left(y^{\prime}, t\right)+\epsilon^{2} \widetilde{V}_{1}^{2}\left(y^{\prime}, t\right) \\
\widetilde{W}_{2,1}^{\mathrm{apr}}\left(y^{\prime}, t\right)= & \widetilde{W}_{2,1}^{0}\left(y^{\prime}, t\right)+\epsilon \widetilde{W}_{2,1}^{1}\left(y^{\prime}, t\right) \\
& +\epsilon^{2} \widetilde{W}_{2,1}^{2}\left(y^{\prime}, t\right) \\
\widetilde{W}_{3,1}^{\mathrm{apr}}\left(y^{\prime}, t\right)= & \widetilde{W}_{3,1}^{0}\left(y^{\prime}, t\right)+\epsilon \widetilde{W}_{3,1}^{1}\left(y^{\prime}, t\right) \\
& +\epsilon^{2} \widetilde{W}_{3,1}^{2}\left(y^{\prime}, t\right) .
\end{aligned}
$$

Due to the small thickness of the pipe, it is reasonable to assume that the functions $\tilde{f}\left(y^{\prime}, t\right), \tilde{g}_{2}\left(y^{\prime}, t\right), \tilde{g}_{3}\left(y^{\prime}, t\right)$ are independent of the cross-section variables $y^{\prime}=$ $\left(y_{2}, y_{3}\right)$. Consequently, we are going to be in position to explicitly compute both zero-order approximation and the correctors.
3.1. Zero-Order Approximation. Plugging (21) and (16d)(16f) into system (20) and collecting the terms of order $\epsilon^{0}=1$, we get

$$
\begin{aligned}
& -\mu \Delta_{y^{\prime}} \widetilde{V}_{1}^{0}\left(y^{\prime}, t\right)=\widetilde{f}^{0}(t), \\
& -\alpha \Delta_{y^{\prime}} \widetilde{W}_{2,1}^{0}\left(y^{\prime}, t\right) \\
& \quad-\beta \frac{\partial}{\partial y_{2}}\left(\frac{\partial \widetilde{W}_{2,1}^{0}\left(y^{\prime}, t\right)}{\partial y_{2}}+\frac{\partial \widetilde{W}_{3,1}^{0}\left(y^{\prime}, t\right)}{\partial y_{3}}\right) \\
& \quad=\widetilde{g}_{2}^{0}(t), \\
& -\alpha \Delta_{y^{\prime}} \widetilde{W}_{3,1}^{0}\left(y^{\prime}, t\right) \\
& \quad-\beta \frac{\partial}{\partial y_{3}}\left(\frac{\partial \widetilde{W}_{2,1}^{0}\left(y^{\prime}, t\right)}{\partial y_{2}}+\frac{\partial \widetilde{W}_{3,1}^{0}\left(y^{\prime}, t\right)}{\partial y_{3}}\right) \\
& \quad=\widetilde{g}_{3}^{0}(t), \\
& \left.\widetilde{V}_{1}^{0}\left(y^{\prime}, t\right)\right|_{\partial B}=0, \\
& \left.\widetilde{W}_{2,1}^{0}\left(y^{\prime}, t\right)\right|_{\partial B}=0, \\
& \left.\widetilde{W}_{3,1}^{0}\left(y^{\prime}, t\right)\right|_{\partial B}=0 .
\end{aligned}
$$

Note that the problems for the velocity and microrotation are decoupled at this particular stage. Equation (22a) with the boundary condition (22d) can be solved by taking

$$
\widetilde{V}_{1}^{0}\left(y^{\prime}, t\right)=\frac{1}{\mu} \chi_{0}\left(y^{\prime}\right) \widetilde{f}^{0}(t),
$$

where $\chi_{0}\left(y^{\prime}\right)$ denotes the solution of the auxiliary problem posed on $B$ :

$$
\begin{aligned}
-\Delta_{y^{\prime}} \chi_{0}\left(y^{\prime}\right) & =1, \quad y^{\prime} \in B, \\
\left.\chi_{0}\left(y^{\prime}\right)\right|_{\partial B} & =0 .
\end{aligned}
$$

Since we assumed the pipe has a circular cross-section, namely,

$$
B=\left\{y^{\prime} \in \mathbb{R}^{2}:\left|y^{\prime}\right|<R\right\}, \quad R>0,
$$

we can pass to polar coordinates $(r, \theta)$ and compute $\chi_{0}$ explicitly from (24):

$$
\chi_{0}\left(y^{\prime}\right)=\frac{1}{4}\left(R^{2}-\left|y^{\prime}\right|^{2}\right)
$$

The explicit expression for the zero-order velocity approximation $\widetilde{V}_{1}^{0}\left(y^{\prime}, t\right)$ now reads

$$
\widetilde{V}_{1}^{0}\left(y^{\prime}, t\right)=\frac{1}{4 \mu}\left(R^{2}-\left|y^{\prime}\right|^{2}\right) \widetilde{f}^{0}(t) .
$$


Similarly, it can be straightforwardly verified that problem (22b), (22c) with the boundary conditions (22e)-(22f) for microrotation will be satisfied for

$$
\begin{aligned}
\widetilde{W}_{2,1}^{0}\left(y^{\prime}, t\right) & =\frac{2}{2 \alpha+\beta} \chi_{0}\left(y^{\prime}\right) \widetilde{g}_{2}^{0}(t) \\
& =\frac{1}{2(2 \alpha+\beta)}\left(R^{2}-\left|y^{\prime}\right|^{2}\right) \widetilde{g}_{2}^{0}(t), \\
\widetilde{W}_{3,1}^{0}\left(y^{\prime}, t\right) & =\frac{2}{2 \alpha+\beta} \chi_{0}\left(y^{\prime}\right) \widetilde{g}_{3}^{0}(t) \\
& =\frac{1}{2(2 \alpha+\beta)}\left(R^{2}-\left|y^{\prime}\right|^{2}\right) \widetilde{g}_{3}^{0}(t) .
\end{aligned}
$$

3.2. First-Order Corrector. Now, we compute the firstorder corrector $\left(\widetilde{V}_{1}^{1}\left(y^{\prime}, t\right), \widetilde{W}_{2,1}^{1}\left(y^{\prime}, t\right), \widetilde{W}_{3,1}^{1}\left(y^{\prime}, t\right)\right)$. Inserting (21) and (16d)-(16f) into the system of equations (20), after collecting the terms of order $\epsilon$, we obtain

$$
\begin{aligned}
& -\mu \Delta_{y^{\prime}} \widetilde{V}_{1}^{1}\left(y^{\prime}, t\right) \\
& =a\left(\frac{\partial \widetilde{W}_{3,1}^{0}\left(y^{\prime}, t\right)}{\partial y_{2}}-\frac{\partial \widetilde{W}_{2,1}^{0}\left(y^{\prime}, t\right)}{\partial y_{3}}\right)+\widetilde{f}^{1}(t) \\
& -\alpha \Delta_{y^{\prime}} \widetilde{W}_{2,1}^{1}\left(y^{\prime}, t\right) \\
& -\beta \frac{\partial}{\partial y_{2}}\left(\frac{\partial \widetilde{W}_{2,1}^{1}\left(y^{\prime}, t\right)}{\partial y_{2}}+\frac{\partial \widetilde{W}_{3,1}^{1}\left(y^{\prime}, t\right)}{\partial y_{3}}\right) \\
& \quad=a \frac{\partial \widetilde{V}_{1}^{0}\left(y^{\prime}, t\right)}{\partial y_{3}}+\widetilde{g}_{2}^{1}(t), \\
& -\alpha \Delta_{y^{\prime}} \widetilde{W}_{3,1}^{1}\left(y^{\prime}, t\right) \\
& -\beta \frac{\partial}{\partial y_{3}}\left(\frac{\partial \widetilde{W}_{2,1}^{1}\left(y^{\prime}, t\right)}{\partial y_{2}}+\frac{\partial \widetilde{W}_{3,1}^{1}\left(y^{\prime}, t\right)}{\partial y_{3}}\right) \\
& \left.\widetilde{W}_{3,1}^{1}\left(y^{\prime}, t\right)\right|_{\partial B}=0 . \\
& \left.\widetilde{W}_{2,1}^{1}\left(y^{\prime}, t\right)\right|_{\partial B}=0, a \widetilde{V}_{1}^{0}\left(y^{\prime}, t\right) \\
& \left.\widetilde{V}_{1}^{1}\left(y^{\prime}, t\right)\right|_{\partial B}=0, \widetilde{g}_{3}^{1}(t),
\end{aligned}
$$

The system is not decoupled anymore, so the effects of the microstructure on the fluid velocity will be present. Using expressions (28a) and (28b) for the microrotation zero-order approximation $\left(\widetilde{W}_{2,1}^{0}\left(y^{\prime}, t\right), \widetilde{W}_{3,1}^{0}\left(y^{\prime}, t\right)\right)$, we get

$$
\begin{aligned}
\mu \Delta_{y^{\prime}} \widetilde{V}_{1}^{1}\left(y^{\prime}, t\right)= & a\left(\frac{\widetilde{g}_{3}^{0}(t)}{2 \alpha+\beta} y_{2}-\frac{\widetilde{g}_{2}^{0}(t)}{2 \alpha+\beta} y_{3}\right) \\
& -\widetilde{f}^{1}(t), \\
\left.\widetilde{V}_{1}^{1}\left(y^{\prime}, t\right)\right|_{\partial B}= & 0 .
\end{aligned}
$$

Let us introduce $\chi_{i}\left(y^{\prime}\right), i=1,2$, as the solutions of the following problems:

$$
\begin{aligned}
& \Delta_{y^{\prime}} \chi_{i}\left(y^{\prime}\right)=y_{i+1}, \quad y^{\prime} \in B, \\
& \left.\chi_{i}\left(y^{\prime}\right)\right|_{\partial B}=0 .
\end{aligned}
$$

Taking into account (25) and passing to the polar coordinates easily give

$$
\begin{aligned}
& \chi_{1}\left(y^{\prime}\right)=\frac{1}{8}\left(\left|y^{\prime}\right|^{2}-R^{2}\right) y_{2}, \\
& \chi_{2}\left(y^{\prime}\right)=\frac{1}{8}\left(\left|y^{\prime}\right|^{2}-R^{2}\right) y_{3} .
\end{aligned}
$$

We seek for the solution of system (30) in the form

$$
\begin{aligned}
& \widetilde{V}_{1}^{1}\left(y^{\prime}, t\right)=\frac{1}{\mu}\left[\frac{a \widetilde{g}_{3}^{0}(t)}{2 \alpha+\beta} \chi_{1}\left(y^{\prime}\right)-\frac{a \widetilde{g}_{2}^{0}(t)}{2 \alpha+\beta} \chi_{2}\left(y^{\prime}\right)\right. \\
& \left.\quad+\chi_{0}\left(y^{\prime}\right) \widetilde{f}^{1}(t)\right]
\end{aligned}
$$

leading to

$$
\begin{aligned}
\widetilde{V}_{1}^{1}\left(y^{\prime}, t\right)= & \frac{1}{8 \mu}\left(\left|y^{\prime}\right|^{2}-R^{2}\right) \\
& \cdot\left[\frac{a \widetilde{g}_{3}^{0}(t)}{2 \alpha+\beta} y_{2}-\frac{a \widetilde{g}_{2}^{0}(t)}{2 \alpha+\beta} y_{3}-2 \widetilde{f}^{1}(t)\right] .
\end{aligned}
$$

Now we compute the corrector for the microrotation. Plugging expression (27) for $\widetilde{V}_{1}^{0}\left(y^{\prime}, t\right)$ into (29) yields

$$
\begin{aligned}
& \alpha \Delta_{y^{\prime}} \widetilde{W}_{2,1}^{1}\left(y^{\prime}, t\right) \\
& +\beta \frac{\partial}{\partial y_{2}}\left(\frac{\partial \widetilde{W}_{2,1}^{1}\left(y^{\prime}, t\right)}{\partial y_{2}}+\frac{\partial \widetilde{W}_{3,1}^{1}\left(y^{\prime}, t\right)}{\partial y_{3}}\right) \\
& =\frac{a \widetilde{f}^{0}(t)}{2 \mu} y_{3}-\tilde{g}_{2}^{1}(t), \\
& \alpha \Delta_{y^{\prime}} \widetilde{W}_{3,1}^{1}\left(y^{\prime}, t\right) \\
& +\beta \frac{\partial}{\partial y_{3}}\left(\frac{\partial \widetilde{W}_{2,1}^{1}\left(y^{\prime}, t\right)}{\partial y_{2}}+\frac{\partial \widetilde{W}_{3,1}^{1}\left(y^{\prime}, t\right)}{\partial y_{3}}\right) \\
& =-\frac{a \tilde{f}^{0}(t)}{2 \mu} y_{2}-\tilde{g}_{3}^{1}(t), \\
& \left.\widetilde{W}_{2,1}^{1}\left(y^{\prime}, t\right)\right|_{\partial B}=0 \text {, } \\
& \left.\widetilde{W}_{3,1}^{1}\left(y^{\prime}, t\right)\right|_{\partial B}=0 \text {. }
\end{aligned}
$$


Similarly, as for $\widetilde{V}_{1}^{1}\left(y^{\prime}, t\right)$, we obtain explicit expression for $\left(\widetilde{W}_{2,1}^{1}\left(y^{\prime}, t\right), \widetilde{W}_{3,1}^{1}\left(y^{\prime}, t\right)\right)$; namely,

$$
\begin{aligned}
& \widetilde{W}_{2,1}^{1}\left(y^{\prime}, t\right)=\left(\left|y^{\prime}\right|^{2}-R^{2}\right) \\
& \cdot\left[y_{3} \frac{a}{16 \mu \alpha} \widetilde{f}^{0}(t)-\frac{1}{2(2 \alpha+\beta)} \widetilde{g}_{2}^{1}(t)\right], \\
& \widetilde{W}_{3,1}^{1}\left(y^{\prime}, t\right)=\left(\left|y^{\prime}\right|^{2}-R^{2}\right) \\
& \cdot\left[-y_{2} \frac{a}{16 \mu \alpha} \widetilde{f}^{0}(t)-\frac{1}{2(2 \alpha+\beta)} \widetilde{g}_{3}^{1}(t)\right] .
\end{aligned}
$$

3.3. Second-Order Corrector. To capture the effects of the time derivative as well, we have to look for the second-order corrector. Substituting (21) and (16d)-(16f) into system (20) and collecting the terms of order $\epsilon^{2}$ yield

$$
\begin{aligned}
& -\mu \Delta_{y^{\prime}} \widetilde{V}_{1}^{2}\left(y^{\prime}, t\right) \\
& =a\left(\frac{\partial \widetilde{W}_{3,1}^{1}\left(y^{\prime}, t\right)}{\partial y_{2}}-\frac{\partial \widetilde{W}_{2,1}^{1}\left(y^{\prime}, t\right)}{\partial y_{3}}\right)+\widetilde{f}^{2}(t) \\
& -\frac{\partial \widetilde{V}_{1}^{0}\left(y^{\prime}, t\right)}{\partial t} \\
& -\alpha \Delta_{y^{\prime}} \widetilde{W}_{2,1}^{2}\left(y^{\prime}, t\right) \\
& -\beta \frac{\partial}{\partial y_{2}}\left(\frac{\partial \widetilde{W}_{2,1}^{2}\left(y^{\prime}, t\right)}{\partial y_{2}}+\frac{\partial \widetilde{W}_{3,1}^{2}\left(y^{\prime}, t\right)}{\partial y_{3}}\right) \\
& =a \frac{\partial \widetilde{V}_{1}^{1}\left(y^{\prime}, t\right)}{\partial y_{3}}+\widetilde{g}_{2}^{2}(t)-\frac{\partial \widetilde{W}_{2,1}^{0}\left(y^{\prime}, t\right)}{\partial t} \\
& -2 a \widetilde{W}_{2,1}^{0}\left(y^{\prime}, t\right), \\
& -\alpha \Delta_{y^{\prime}} \widetilde{W}_{3,1}^{2}\left(y^{\prime}, t\right) \\
& -\beta \frac{\partial}{\partial y_{3}}\left(\frac{\partial \widetilde{W}_{2,1}^{2}\left(y^{\prime}, t\right)}{\partial y_{2}}+\frac{\partial \widetilde{W}_{3,1}^{2}\left(y^{\prime}, t\right)}{\partial y_{3}}\right) \\
& =-a \frac{\partial \widetilde{V}_{1}^{1}\left(y^{\prime}, t\right)}{\partial y_{2}}+\widetilde{g}_{3}^{2}(t)-\frac{\partial \widetilde{W}_{3,1}^{0}\left(y^{\prime}, t\right)}{\partial t} \\
& -2 a \widetilde{W}_{3,1}^{0}\left(y^{\prime}, t\right), \\
& \left.\widetilde{V}_{1}^{2}\left(y^{\prime}, t\right)\right|_{\partial B}=0, \\
& \left.\widetilde{W}_{2,1}^{2}\left(y^{\prime}, t\right)\right|_{\partial B}=0, \\
& \left.\widetilde{W}_{3,1}^{2}\left(y^{\prime}, t\right)\right|_{\partial B}=0 .
\end{aligned}
$$

Taking into account (27) and (36), we get the following problem for $\widetilde{V}_{1}^{2}\left(y^{\prime}, t\right)$ :

$$
\begin{aligned}
& -\mu \Delta_{y^{\prime}} \widetilde{V}_{1}^{2}\left(y^{\prime}, t\right)=a\left(-\frac{a \widetilde{f}^{0}(t)}{4 \mu \alpha}\left(y_{2}^{2}+y_{3}^{2}-\frac{R^{2}}{2}\right)\right. \\
& \left.-\frac{\widetilde{g}_{3}^{1}(t)}{2 \alpha+\beta} y_{2}-\frac{\widetilde{g}_{2}^{1}(t)}{2 \alpha+\beta} y_{3}\right)+\widetilde{f}^{2}(t)-\left(R^{2}-y_{2}^{2}\right. \\
& \left.-y_{3}^{2}\right) \frac{1}{4 \mu} \frac{d}{d t} \widetilde{f}^{0}(t), \\
& \left.\widetilde{V}_{1}^{2}\left(y^{\prime}, t\right)\right|_{\partial B}=0 .
\end{aligned}
$$

We rewrite

$$
\begin{aligned}
& -\mu \Delta_{y^{\prime}} \widetilde{V}_{1}^{2}\left(y^{\prime}, t\right) \\
& =\frac{-a^{2} \widetilde{f}^{0}(t)+\alpha(d / d t) \widetilde{f}^{0}(t)}{4 \mu \alpha}\left(y_{2}^{2}+y_{3}^{2}\right) \\
& \quad-\frac{a \widetilde{g}_{3}^{1}(t)}{2 \alpha+\beta} y_{2}-\frac{a \widetilde{g}_{2}^{1}(t)}{2 \alpha+\beta} y_{3}+\widetilde{f}^{2}(t) \\
& +\frac{\left(a^{2} \widetilde{f}^{0}(t)-2 \alpha(d / d t) \widetilde{f}^{0}(t)\right) R^{2}}{8 \mu \alpha}, \\
& \left.\widetilde{V}_{1}^{2}\left(y^{\prime}, t\right)\right|_{\partial в}=0
\end{aligned}
$$

and introduce $\chi_{3}\left(y^{\prime}\right)$ as the solution of the following problem:

$$
\begin{aligned}
& \Delta_{y^{\prime}} \chi_{3}\left(y^{\prime}\right)=y_{2}^{2}+y_{3}^{2}, \quad y^{\prime} \in B, \\
& \left.\chi_{3}\left(y^{\prime}\right)\right|_{\partial B}=0 .
\end{aligned}
$$

Passing to the polar coordinates provides

$$
\chi_{3}\left(y^{\prime}\right)=\frac{1}{16}\left(\left|y^{\prime}\right|^{4}-R^{4}\right) .
$$

We seek for the solution of (39) in the form

$$
\begin{aligned}
\widetilde{V}_{1}^{2}\left(y^{\prime}, t\right) & \\
= & -\frac{1}{\mu} \frac{-a^{2} \widetilde{f}^{0}(t)+\alpha(d / d t) \tilde{f}^{0}(t)}{4 \mu \alpha} \chi_{3}\left(y^{\prime}\right) \\
& +\frac{1}{\mu} \frac{a \widetilde{g}_{3}^{1}(t)}{2 \alpha+\beta} \chi_{1}\left(y^{\prime}\right)+\frac{1}{\mu} \frac{a \widetilde{g}_{2}^{1}(t)}{2 \alpha+\beta} \chi_{2}\left(y^{\prime}\right) \\
& +\frac{1}{\mu} \chi_{0}\left(y^{\prime}\right) \widetilde{f}^{2}(t) \\
& +\frac{R^{2}}{\mu} \frac{a^{2} \widetilde{f}^{0}(t)-2 \alpha(d / d t) \tilde{f}^{0}(t)}{8 \mu \alpha} \chi_{0}\left(y^{\prime}\right),
\end{aligned}
$$


implying

$$
\begin{aligned}
& \widetilde{V}_{1}^{2}\left(y^{\prime}, t\right)=\frac{a^{2} \tilde{f}^{0}(t)-\alpha(d / d t) \tilde{f}^{0}(t)}{64 \mu^{2} \alpha}\left(\left|y^{\prime}\right|^{4}-R^{4}\right) \\
& +\left(\frac{a \widetilde{g}_{3}^{1}(t)}{8 \mu(2 \alpha+\beta)} y_{2}+\frac{a \widetilde{g}_{2}^{1}(t)}{8 \mu(2 \alpha+\beta)} y_{3}\right)\left(\left|y^{\prime}\right|^{2}-R^{2}\right) \\
& +\left(\frac{\tilde{f}^{2}(t)}{4 \mu}+\frac{\left(a^{2} \widetilde{f}^{0}(t)-2 \alpha(d / d t) \tilde{f}^{0}(t)\right) R^{2}}{32 \mu^{2} \alpha}\right) \\
& +\left(R^{2}-\left|y^{\prime}\right|^{2}\right) .
\end{aligned}
$$

Plugging (28a)-(28b) and (34) into (37b) and (37c), we get the following system for the second-order microrotation corrector $\left(\widetilde{W}_{2,1}^{2}\left(y^{\prime}, t\right), \widetilde{W}_{3,1}^{2}\left(y^{\prime}, t\right)\right)$ :

$$
\begin{aligned}
& -\alpha \Delta_{y^{\prime}} \widetilde{W}_{2,1}^{2}\left(y^{\prime}, t\right)-\beta \frac{\partial}{\partial y_{2}}\left(\frac{\partial \widetilde{W}_{2,1}^{2}\left(y^{\prime}, t\right)}{\partial y_{2}}\right. \\
& \left.+\frac{\partial \widetilde{W}_{3,1}^{2}\left(y^{\prime}, t\right)}{\partial y_{3}}\right)=a\left(-\frac{a \widetilde{g}_{2}^{0}(t)}{8 \mu(2 \alpha+\beta)}\left(y_{2}^{2}+3 y_{3}^{2}\right)\right. \\
& \left.+\frac{a \widetilde{g}_{3}^{0}(t)}{4 \mu(2 \alpha+\beta)} y_{2} y_{3}+\frac{\widetilde{f}^{1}(t)}{2 \mu} y_{3}+\frac{a R^{2} \widetilde{g}_{2}^{0}(t)}{8 \mu(2 \alpha+\beta)}\right) \\
& +\widetilde{g}_{2}^{2}(t)+\frac{1}{2(2 \alpha+\beta)}\left(y_{2}^{2}+y_{3}^{2}-R^{2}\right) \frac{d}{d t} \widetilde{g}_{2}^{0}(t)
\end{aligned}
$$

$$
+\frac{a}{2 \alpha+\beta}\left(y_{2}^{2}+y_{3}^{2}-R^{2}\right) \widetilde{g}_{2}^{0}(t),
$$$$
-\alpha \Delta_{y^{\prime}} \widetilde{W}_{3,1}^{2}\left(y^{\prime}, t\right)-\beta \frac{\partial}{\partial y_{3}}\left(\frac{\partial \widetilde{W}_{2,1}^{2}\left(y^{\prime}, t\right)}{\partial y_{2}}\right.
$$$$
\left.+\frac{\partial \widetilde{W}_{3,1}^{2}\left(y^{\prime}, t\right)}{\partial y_{3}}\right)=a\left(-\frac{a \widetilde{g}_{3}^{0}(t)}{8 \mu(2 \alpha+\beta)}\left(3 y_{2}^{2}+y_{3}^{2}\right)\right.
$$$$
\left.+\frac{a \widetilde{g}_{2}^{0}(t)}{4 \mu(2 \alpha+\beta)} y_{2} y_{3}+\frac{\widetilde{f}^{1}(t)}{2 \mu} y_{2}+\frac{a R^{2} \widetilde{g}_{3}^{0}(t)}{8 \mu(2 \alpha+\beta)}\right)
$$$$
+\widetilde{g}_{3}^{2}(t)+\frac{1}{2(2 \alpha+\beta)}\left(y_{2}^{2}+y_{3}^{2}-R^{2}\right) \frac{d}{d t} \widetilde{g}_{3}^{0}(t)
$$$$
+\frac{a}{2 \alpha+\beta}\left(y_{2}^{2}+y_{3}^{2}-R^{2}\right) \widetilde{g}_{3}^{0}(t),
$$

$$
\left.\widetilde{W}_{2,1}^{2}\left(y^{\prime}, t\right)\right|_{\partial B}=0,
$$

$$
\left.\widetilde{W}_{3,1}^{2}\left(y^{\prime}, t\right)\right|_{\partial B}=0 .
$$

After rewriting, we obtain

$$
\begin{aligned}
& -\alpha \Delta_{y^{\prime}} \widetilde{W}_{2,1}^{2}\left(y^{\prime}, t\right)-\beta \frac{\partial}{\partial y_{2}}\left(\frac{\partial \widetilde{W}_{2,1}^{2}\left(y^{\prime}, t\right)}{\partial y_{2}}+\frac{\partial \widetilde{W}_{3,1}^{2}\left(y^{\prime}, t\right)}{\partial y_{3}}\right)=\frac{-a^{2} \widetilde{g}_{2}^{0}(t)+4 \mu(d / d t) \widetilde{g}_{2}^{0}(t)+8 a \mu \widetilde{g}_{2}^{0}(t)}{8 \mu(2 \alpha+\beta)} y_{2}^{2} \\
& +\frac{-3 a^{2} \widetilde{g}_{2}^{0}(t)+4 \mu(d / d t) \widetilde{g}_{2}^{0}(t)+8 a \mu \widetilde{g}_{2}^{0}(t)}{8 \mu(2 \alpha+\beta)} y_{3}^{2}+\frac{a^{2} \widetilde{g}_{3}^{0}(t)}{4 \mu(2 \alpha+\beta)} y_{2} y_{3}+\frac{a \widetilde{f}^{1}(t)}{2 \mu} y_{3} \\
& +\frac{a^{2} R^{2} \widetilde{g}_{2}^{0}(t)+8 \mu(2 \alpha+\beta) \widetilde{g}_{2}^{2}(t)-4 R^{2} \mu(d / d t) \widetilde{g}_{2}^{0}(t)-8 a \mu R^{2} \widetilde{g}_{2}^{0}(t)}{8 \mu(2 \alpha+\beta)}, \\
& -\alpha \Delta_{y^{\prime}} \widetilde{W}_{3,1}^{2}\left(y^{\prime}, t\right)-\beta \frac{\partial}{\partial y_{3}}\left(\frac{\partial \widetilde{W}_{2,1}^{2}\left(y^{\prime}, t\right)}{\partial y_{2}}+\frac{\partial \widetilde{W}_{3,1}^{2}\left(y^{\prime}, t\right)}{\partial y_{3}}\right)=\frac{-3 a^{2} \widetilde{g}_{3}^{0}(t)+4 \mu(d / d t) \widetilde{g}_{3}^{0}(t)+8 a \mu \widetilde{g}_{3}^{0}(t)}{8 \mu(2 \alpha+\beta)} y_{2}^{2} \\
& +\frac{-a^{2} \widetilde{g}_{3}^{0}(t)+4 \mu(d / d t) \widetilde{g}_{3}^{0}(t)+8 a \mu \widetilde{g}_{3}^{0}(t)}{8 \mu(2 \alpha+\beta)} y_{3}^{2}+\frac{a^{2} \widetilde{g}_{2}^{0}(t)}{4 \mu(2 \alpha+\beta)} y_{2} y_{3}+\frac{a \widetilde{f}^{1}(t)}{2 \mu} y_{2} \\
& +\frac{a^{2} R^{2} \widetilde{g}_{3}^{0}(t)+8 \mu(2 \alpha+\beta) \widetilde{g}_{3}^{2}(t)-4 R^{2} \mu(d / d t) \widetilde{g}_{3}^{0}(t)-8 a \mu R^{2} \widetilde{g}_{3}^{0}(t)}{8 \mu(2 \alpha+\beta)}, \\
& \left.\widetilde{W}_{2,1}^{2}\left(y^{\prime}, t\right)\right|_{\partial B}=0 \\
& \left.\widetilde{W}_{3,1}^{2}\left(y^{\prime}, t\right)\right|_{\partial B}=0 .
\end{aligned}
$$

Tedious but straightforward calculation gives

$$
\begin{aligned}
& \widetilde{W}_{2,1}^{2}\left(y^{\prime}, t\right)=\left(\left|y^{\prime}\right|^{2}-R^{2}\right)\left[C_{1} y_{2}^{2}+C_{2} y_{2} y_{3}+C_{3} y_{3}^{2}\right. \\
& \left.\quad+C_{4} y_{2}+C_{5} y_{3}+C_{6}\right],
\end{aligned}
$$$$
\widetilde{W}_{3,1}^{2}\left(y^{\prime}, t\right)=\left(\left|y^{\prime}\right|^{2}-R^{2}\right)\left[C_{7} y_{2}^{2}+C_{8} y_{2} y_{3}+C_{9} y_{3}^{2}\right.
$$$$
\left.+C_{10} y_{2}+C_{11} y_{3}+C_{12}\right] \text {, }
$$ 
where

$$
\begin{aligned}
& C_{1}=\frac{\left(2 \alpha-12 \beta^{2} /(12 \alpha+6 \beta)\right) C_{3}+A-3 \beta H /(12 \alpha+6 \beta)}{-14 \alpha-12 \beta+12 \beta^{2} /(12 \alpha+6 \beta)}, \\
& C_{2}=\frac{-4 \beta\left(C_{7}+C_{9}\right)-C}{12 \alpha+6 \beta}, \\
& C_{3}=\frac{(B-3 \beta H /(12 \alpha+6 \beta))\left(-14 \alpha-12 \beta+12 \beta^{2} /(12 \alpha+6 \beta)\right)-\left(-2 \alpha-2 \beta+12 \beta^{2} /(12 \alpha+6 \beta)\right)(A-3 \beta H /(12 \alpha+6 \beta))}{\left(-2 \alpha-2 \beta+12 \beta^{2} /(12 \alpha+6 \beta)\right)\left(2 \alpha-12 \beta^{2} /(12 \alpha+6 \beta)\right)+\left(-14 \alpha-2 \beta+12 \beta^{2} /(12 \alpha+6 \beta)\right)^{2}}, \\
& C_{4}=0, \\
& C_{5}=\frac{I+(8 \alpha+2 \beta) C_{10}}{-2 \beta}, \\
& C_{6}=\frac{2 \alpha R^{2}\left(C_{1}+C_{3}\right)+2 \beta R^{2} C_{1}+\beta R^{2} C_{8}-E}{4 \alpha+2 \beta}, \\
& C_{7}=\frac{F-3 \beta C /(12 \alpha+6 \beta)+\left(2 \alpha+2 \beta-12 \beta^{2} /(12 \alpha+6 \beta)\right) C_{9}}{-14 \alpha-2 \beta+12 \beta^{2} /(12 \alpha+6 \beta)}, \\
& C_{8}=\frac{-4 \beta\left(C_{1}+C_{3}\right)-H}{12 \alpha+6 \beta}, \\
& C_{9} \\
& =\frac{(G-3 \beta C /(12 \alpha+6 \beta))\left(-14 \alpha-2 \beta+12 \beta^{2} /(12 \alpha+6 \beta)\right)+\left(2 \alpha-12 \beta^{2} /(12 \alpha+6 \beta)\right)(F-3 \beta C /(12 \alpha+6 \beta))}{\left(-2 \alpha+12 \beta^{2} /(12 \alpha+6 \beta)\right)\left(2 \alpha+2 \beta-12 \beta^{2} /(12 \alpha+6 \beta)\right)+\left(-14 \alpha-12 \beta+12 \beta^{2} /(12 \alpha+6 \beta)\right)\left(-14 \alpha-2 \beta+12 \beta^{2} /(12 \alpha+6 \beta)\right)}, \\
& C_{10}=\frac{2 D \beta-I(8 \alpha+2 \beta)}{(8 \alpha+2 \beta)^{2}-4 \beta^{2}}, \\
& C_{11}=0, \\
& C_{12}=\frac{2 \alpha R^{2}\left(C_{7}+C_{9}\right)+2 \beta R^{2} C_{9}+\beta R^{2} C_{2}-J}{4 \alpha+2 \beta},
\end{aligned}
$$

where $A=\left(-a^{2} \widetilde{g}_{2}^{0}(t)+4 \mu(d / d t) \widetilde{g}_{2}^{0}(t)+8 a \mu \widetilde{g}_{2}^{0}(t)\right) / 8 \mu(2 \alpha+$ $\beta), B=\left(-3 a^{2} \widetilde{g}_{2}^{0}(t)+4 \mu(d / d t) \widetilde{g}_{2}^{0}(t)+8 a \mu \widetilde{g}_{2}^{0}(t)\right) / 8 \mu(2 \alpha+\beta)$, $C=a^{2} \widetilde{g}_{3}^{0}(t) / 4 \mu(2 \alpha+\beta), D=a \widetilde{f}^{1}(t) / 2 \mu, E=\left(a^{2} R^{2} \widetilde{g}_{2}^{0}(t)+\right.$ $\left.8 \mu(2 \alpha+\beta) \widetilde{g}_{2}^{2}(t)-4 R^{2} \mu(d / d t) \widetilde{g}_{2}^{0}(t)-8 a \mu R^{2} \widetilde{g}_{2}^{0}(t)\right) / 8 \mu(2 \alpha+$ $\beta), F=\left(-3 a^{2} \widetilde{g}_{3}^{0}(t)+4 \mu(d / d t) \widetilde{g}_{3}^{0}(t)+8 a \mu \widetilde{g}_{3}^{0}(t)\right) / 8 \mu(2 \alpha+\beta)$, $G=\left(-a^{2} \widetilde{g}_{3}^{0}(t)+4 \mu(d / d t) \widetilde{g}_{3}^{0}(t)+8 a \mu \widetilde{g}_{3}^{0}(t)\right) / 8 \mu(2 \alpha+\beta), H=$ $a^{2} \widetilde{g}_{2}^{0}(t) / 4 \mu(2 \alpha+\beta), I=a \widetilde{f}^{1}(t) / 2 \mu, J=\left(a^{2} R^{2} \widetilde{g}_{3}^{0}(t)+8 \mu(2 \alpha+\right.$ $\left.\beta) \widetilde{g}_{3}^{2}(t)-4 R^{2} \mu(d / d t) \widetilde{g}_{3}^{0}(t)-8 a \mu R^{2} \widetilde{g}_{3}^{0}(t)\right) / 8 \mu(2 \alpha+\beta)$.

\section{Micropolar Inverse Problem}

Now, we turn our attention to micropolar inverse problem (12). First, we notice that

$$
\begin{aligned}
H(t) & =\sum_{j=0}^{2} \epsilon^{j+2} F^{j}(t)-\sum_{j=0}^{2} \int_{B^{e}} \epsilon^{j} \widetilde{V}_{1}^{j}\left(\frac{x^{\prime}}{\epsilon}, t\right) d x^{\prime} \\
& =\sum_{j=0}^{2} \epsilon^{j+2} F^{j}(t)-\sum_{j=0}^{2} \int_{B} \epsilon^{j+2} \widetilde{V}_{1}^{j}\left(y^{\prime}, t\right) d y^{\prime} \\
& =\sum_{j=0}^{2} \epsilon^{j+2} H^{j}(t) .
\end{aligned}
$$

Denoting

$$
H^{j}(t)=F^{j}(t)-\int_{B} \widetilde{V}_{1}^{j}\left(y^{\prime}, t\right) d y^{\prime}, \quad j=0,1,2,
$$

we have the compatibility condition (due to (14))

$$
H^{j}(0)=0, \quad j=0,1,2,
$$

or

$$
F^{j}(0)=\int_{B} \widetilde{a}^{j}\left(y^{\prime}\right) d y^{\prime}, \quad j=0,1,2 .
$$

Performing the change of variables $y^{\prime}=x^{\prime} / \epsilon$ and putting $q(t)=\left(1 / \epsilon^{2}\right) s(t)$, from (12) we deduce

$$
\begin{aligned}
- & \mu \Delta_{y^{\prime}} \widetilde{V}_{2}\left(y^{\prime}, t\right) \\
& =a \epsilon\left(\frac{\partial \widetilde{W}_{3,2}\left(y^{\prime}, t\right)}{\partial y_{2}}-\frac{\partial \widetilde{W}_{2,2}\left(y^{\prime}, t\right)}{\partial y_{3}}\right)+s(t)
\end{aligned}
$$




$$
\begin{aligned}
& -\epsilon^{2} \frac{\partial \widetilde{V}_{2}}{\partial t}\left(y^{\prime}, t\right), \\
& -\alpha \Delta_{y^{\prime}} \widetilde{W}_{2,2}\left(y^{\prime}, t\right) \\
& -\beta \frac{\partial}{\partial y_{2}}\left(\frac{\partial \widetilde{W}_{2,2}\left(y^{\prime}, t\right)}{\partial y_{2}}+\frac{\partial \widetilde{W}_{3,2}\left(y^{\prime}, t\right)}{\partial y_{3}}\right) \\
& =a \epsilon \frac{\partial \widetilde{V}_{2}\left(y^{\prime}, t\right)}{\partial y_{3}}-\epsilon^{2} \frac{\partial \widetilde{W}_{2,2}\left(y^{\prime}, t\right)}{\partial t} \\
& -2 a \epsilon^{2} \widetilde{W}_{2,2}\left(y^{\prime}, t\right) \text {, } \\
& -\alpha \Delta_{y^{\prime}} \widetilde{W}_{3,2}\left(y^{\prime}, t\right) \\
& -\beta \frac{\partial}{\partial y_{3}}\left(\frac{\partial \widetilde{W}_{2,2}\left(y^{\prime}, t\right)}{\partial y_{2}}+\frac{\partial \widetilde{W}_{3,2}\left(y^{\prime}, t\right)}{\partial y_{3}}\right) \\
& =-a \epsilon \frac{\partial \widetilde{V}_{2}\left(y^{\prime}, t\right)}{\partial y_{2}}-\epsilon^{2} \frac{\partial \widetilde{W}_{3,2}\left(y^{\prime}, t\right)}{\partial t} \\
& -2 a \epsilon^{2} \widetilde{W}_{3,2}\left(y^{\prime}, t\right), \\
& \left.\widetilde{V}_{2}\left(y^{\prime}, t\right)\right|_{\partial B}=0, \\
& \left.\widetilde{W}_{2,2}\left(y^{\prime}, t\right)\right|_{\partial B}=0 \text {, } \\
& \left.\widetilde{W}_{3,2}\left(y^{\prime}, t\right)\right|_{\partial B}=0 \text {, } \\
& \widetilde{V}_{2}\left(y^{\prime}, 0\right)=0 \text {, } \\
& \widetilde{W}_{2,2}\left(y^{\prime}, 0\right)=0 \text {, } \\
& \widetilde{W}_{3,2}\left(y^{\prime}, 0\right)=0 \text {, } \\
& \int_{B} \widetilde{V}_{2}\left(y^{\prime}, t\right) d y^{\prime}=\frac{1}{\epsilon^{2}} H(t),
\end{aligned}
$$

where $\widetilde{V}_{2}\left(y^{\prime}, t\right)=V_{2}^{\epsilon}\left(\epsilon y^{\prime}, t\right), \widetilde{W}_{2,2}\left(y^{\prime}, t\right)=W_{2,2}^{\epsilon}\left(\epsilon y^{\prime}, t\right)$, $\widetilde{W}_{3,2}\left(y^{\prime}, t\right)=W_{3,2}^{\epsilon}\left(\epsilon y^{\prime}, t\right)$. In the sequel, we look for the asymptotic approximation in the form

$$
\begin{aligned}
\widetilde{V}_{2}^{\mathrm{apr}}\left(y^{\prime}, t\right)= & \widetilde{V}_{2}^{0}\left(y^{\prime}, t\right)+\epsilon \widetilde{V}_{2}^{1}\left(y^{\prime}, t\right)+\epsilon^{2} \widetilde{V}_{2}^{2}\left(y^{\prime}, t\right), \\
\widetilde{W}_{2,2}^{\mathrm{apr}}\left(y^{\prime}, t\right)= & \widetilde{W}_{2,2}^{0}\left(y^{\prime}, t\right)+\epsilon \widetilde{W}_{2,2}^{1}\left(y^{\prime}, t\right) \\
& +\epsilon^{2} \widetilde{W}_{2,2}^{2}\left(y^{\prime}, t\right) \\
\widetilde{W}_{3,2}^{\mathrm{apr}}\left(y^{\prime}, t\right)= & \widetilde{W}_{3,2}^{0}\left(y^{\prime}, t\right)+\epsilon \widetilde{W}_{3,2}^{1}\left(y^{\prime}, t\right) \\
& +\epsilon^{2} \widetilde{W}_{3,2}^{2}\left(y^{\prime}, t\right) \\
s^{\mathrm{apr}}(t)= & s^{0}(t)+\epsilon s^{1}(t)+\epsilon^{2} s^{2}(t) .
\end{aligned}
$$

4.1. Zero-Order Approximation. For the zero-order approximation $\left(\widetilde{V}_{2}^{0}\left(y^{\prime}, t\right), \widetilde{W}_{2,2}^{0}\left(y^{\prime}, t\right), \widetilde{W}_{3,2}^{0}\left(y^{\prime}, t\right), s^{0}(t)\right)$, we obtain the following problem:

$$
\begin{aligned}
& -\mu \Delta_{y^{\prime}} \widetilde{V}_{2}^{0}\left(y^{\prime}, t\right)=s^{0}(t), \\
& -\alpha \Delta_{y^{\prime}} \widetilde{W}_{2,2}^{0}\left(y^{\prime}, t\right) \\
& \quad-\beta \frac{\partial}{\partial y_{2}}\left(\frac{\partial \widetilde{W}_{2,2}^{0}\left(y^{\prime}, t\right)}{\partial y_{2}}+\frac{\partial \widetilde{W}_{3,2}^{0}\left(y^{\prime}, t\right)}{\partial y_{3}}\right)=0, \\
& -\alpha \Delta_{y^{\prime}} \widetilde{W}_{3,2}^{0}\left(y^{\prime}, t\right) \\
& \quad-\beta \frac{\partial}{\partial y_{3}}\left(\frac{\partial \widetilde{W}_{2,2}^{0}\left(y^{\prime}, t\right)}{\partial y_{2}}+\frac{\partial \widetilde{W}_{3,2}^{0}\left(y^{\prime}, t\right)}{\partial y_{3}}\right)=0, \\
& \left.\widetilde{V}_{2}^{0}\left(y^{\prime}, t\right)\right|_{\partial B}=0, \\
& \left.\widetilde{W}_{2,2}^{0}\left(y^{\prime}, t\right)\right|_{\partial B}=0, \\
& \left.\widetilde{W}_{3,2}^{0}\left(y^{\prime}, t\right)\right|_{\partial B}=0, \\
& \int_{B} \widetilde{V}_{2}^{0}\left(y^{\prime}, t\right) d y^{\prime}=H^{0}(t),
\end{aligned}
$$

where $H^{0}(t)$ is given by

$$
\begin{aligned}
H^{0}(t) & =F^{0}(t)-\int_{B} \widetilde{V}_{1}^{0}\left(y^{\prime}, t\right) d y^{\prime} \\
& =F^{0}(t)-\frac{R^{4} \pi}{8 \mu} \widetilde{f}^{0}(t) .
\end{aligned}
$$

Equation (54a) with the boundary condition (54d) and the flux rate $(54 \mathrm{~g})$ can be solved by taking

$$
\widetilde{V}_{2}^{0}\left(y^{\prime}, t\right)=\frac{1}{4 \mu}\left(R^{2}-\left|y^{\prime}\right|^{2}\right) s^{0}(t) .
$$

Here we choose $s^{0}(t)$ to satisfy the flux condition:

$$
s^{0}(t)=\kappa_{0}^{-1} H^{0}(t)
$$

where

$$
\kappa_{0}=\int_{B} \frac{1}{4 \mu}\left(R^{2}-\left|y^{\prime}\right|^{2}\right)=\frac{R^{4} \pi}{8 \mu}>0 .
$$

We now look at the system (54b), (54c), (54e)-(54f) and observe it has the same structure as the system (22b), (22c), (22e)-(22f) for the zero-order approximation of the micropolar heat problem with $\left(\widetilde{g}_{2}^{0}(t), \widetilde{g}_{3}^{0}(t)\right) \equiv(0,0)$ (see Section 3.1). Thus, we conclude

$$
\left(\widetilde{W}_{2,2}^{0}\left(y^{\prime}, t\right), \widetilde{W}_{3,2}^{0}\left(y^{\prime}, t\right)\right) \equiv(0,0) .
$$


4.2. First-Order Corrector. The first-order corrector $\left(\widetilde{V}_{2}^{1}\left(y^{\prime}, t\right)\right.$, $\left.\widetilde{W}_{2,2}^{1}\left(y^{\prime}, t\right), \widetilde{W}_{3,2}^{1}\left(y^{\prime}, t\right), s^{1}(t)\right)$ is given by the following problem:

$$
\begin{aligned}
& -\mu \Delta_{y^{\prime}} \widetilde{V}_{2}^{1}\left(y^{\prime}, t\right) \\
& =a\left(\frac{\partial \widetilde{W}_{3,2}^{0}\left(y^{\prime}, t\right)}{\partial y_{2}}-\frac{\partial \widetilde{W}_{2,2}^{0}\left(y^{\prime}, t\right)}{\partial y_{3}}\right)+s^{1}(t), \\
& -\alpha \Delta_{y^{\prime}} \widetilde{W}_{2,2}^{1}\left(y^{\prime}, t\right) \\
& -\beta \frac{\partial}{\partial y_{2}}\left(\frac{\partial \widetilde{W}_{2,2}^{1}\left(y^{\prime}, t\right)}{\partial y_{2}}+\frac{\partial \widetilde{W}_{3,2}^{1}\left(y^{\prime}, t\right)}{\partial y_{3}}\right) \\
& =a \frac{\partial \widetilde{V}_{2}^{0}\left(y^{\prime}, t\right)}{\partial y_{3}} \\
& -\alpha \Delta \widetilde{y}^{\prime} \widetilde{W}_{3,2}^{1}\left(y^{\prime}, t\right) \\
& -\beta \frac{\partial}{\partial y_{3}}\left(\frac{\partial \widetilde{W}_{2,2}^{1}\left(y^{\prime}, t\right)}{\partial y_{2}}+\frac{\partial \widetilde{W}_{3,2}^{1}\left(y^{\prime}, t\right)}{\partial y_{3}}\right) \\
& =-a \frac{\partial \widetilde{V}_{2}^{0}\left(y^{\prime}, t\right)}{\partial y_{2}}, \\
& \left.\widetilde{V}_{2}^{1}\left(y^{\prime}, t\right)\right|_{\partial B}=0, \\
& \left.\widetilde{W}_{2,2}^{1}\left(y^{\prime}, t\right)\right|_{\partial B}=0 \\
& \left.\widetilde{W}_{3,2}^{1}\left(y^{\prime}, t\right)\right|_{\partial B}=0 \\
& \int_{B} \widetilde{V}_{2}^{1}\left(y^{\prime}, t\right) d y^{\prime}=H^{1}(t) \\
& \quad
\end{aligned}
$$

where $H^{1}(t)$ is given by

$$
\begin{aligned}
H^{1}(t) & =F^{1}(t)-\int_{B} \widetilde{V}_{1}^{1}\left(y^{\prime}, t\right) d y^{\prime} \\
& =F^{1}(t)-\frac{R^{4} \pi}{8 \mu} \widetilde{f}^{1}(t) .
\end{aligned}
$$

We notice that the system is not decoupled anymore. Inserting the expression for $\left(\widetilde{V}_{2}^{0}\left(y^{\prime}, t\right), \widetilde{W}_{2,2}^{0}\left(y^{\prime}, t\right), \widetilde{W}_{3,2}^{0}\left(y^{\prime}, t\right)\right)$ given by (56) and (59), we obtain

$$
\begin{aligned}
& -\mu \Delta_{y^{\prime}} \widetilde{V}_{2}^{1}\left(y^{\prime}, t\right)=s^{1}(t), \\
& -\alpha \Delta_{y^{\prime}} \widetilde{W}_{2,2}^{1}\left(y^{\prime}, t\right) \\
& -\beta \frac{\partial}{\partial y_{2}}\left(\frac{\partial \widetilde{W}_{2,2}^{1}\left(y^{\prime}, t\right)}{\partial y_{2}}+\frac{\partial \widetilde{W}_{3,2}^{1}\left(y^{\prime}, t\right)}{\partial y_{3}}\right) \\
& =-\frac{a}{2 \mu} y_{3} s^{0}(t),
\end{aligned}
$$

$$
\begin{aligned}
& -\alpha \Delta_{y^{\prime}} \widetilde{W}_{3,2}^{1}\left(y^{\prime}, t\right) \\
& \quad-\beta \frac{\partial}{\partial y_{3}}\left(\frac{\partial \widetilde{W}_{2,2}^{1}\left(y^{\prime}, t\right)}{\partial y_{2}}+\frac{\partial \widetilde{W}_{3,2}^{1}\left(y^{\prime}, t\right)}{\partial y_{3}}\right) \\
& \quad=\frac{a}{2 \mu} y_{2} s^{0}(t) \\
& \left.\widetilde{V}_{2}^{1}\left(y^{\prime}, t\right)\right|_{\partial B}=0 \\
& \left.\widetilde{W}_{2,2}^{1}\left(y^{\prime}, t\right)\right|_{\partial B}=0 \\
& \left.\widetilde{W}_{3,2}^{1}\left(y^{\prime}, t\right)\right|_{\partial B}=0, \\
& \int_{B} \widetilde{V}_{2}^{1}\left(y^{\prime}, t\right) d y^{\prime}=H^{1}(t) .
\end{aligned}
$$

As above, (60a) with the boundary condition (60d) and flux rate $(60 \mathrm{~g})$ can be solved by taking

$$
\widetilde{V}_{2}^{1}\left(y^{\prime}, t\right)=\frac{1}{4 \mu}\left(R^{2}-\left|y^{\prime}\right|^{2}\right) s^{1}(t),
$$

with $s^{1}(t)$ obeying the flux condition:

$$
s^{1}(t)=\kappa_{1}^{-1} H^{1}(t)
$$

with

$$
\kappa_{1}=\int_{B} \frac{1}{4 \mu}\left(R^{2}-\left|y^{\prime}\right|^{2}\right)=\frac{R^{4} \pi}{8 \mu}>0 .
$$

One can easily verify that the system (62b), (62c) for the microrotation corrector with the boundary conditions (62e)(62f) is satisfied for

$$
\begin{aligned}
& \widetilde{W}_{2,2}^{1}\left(y^{\prime}, t\right)=\left(\left|y^{\prime}\right|^{2}-R^{2}\right) \frac{a H^{0}(t)}{16 \mu \alpha \kappa_{0}} y_{3}, \\
& \widetilde{W}_{3,2}^{1}\left(y^{\prime}, t\right)=\left(\left|y^{\prime}\right|^{2}-R^{2}\right) \frac{-a H^{0}(t)}{16 \mu \alpha \kappa_{0}} y_{2} .
\end{aligned}
$$

4.3. Second-Order Corrector. To conclude the analysis for the micropolar inverse problem (52), we identify the terms of order $\epsilon^{2}$ to get the system for the second-order corrector $\left(\widetilde{V}_{2}^{2}\left(y^{\prime}, t\right), \widetilde{W}_{2,2}^{2}\left(y^{\prime}, t\right), \widetilde{W}_{3,2}^{2}\left(y^{\prime}, t\right), s^{2}(t)\right)$. It reads

$$
\begin{aligned}
-\mu \Delta_{y^{\prime}} \widetilde{V}_{2}^{2}\left(y^{\prime}, t\right) & \\
& =a\left(\frac{\partial \widetilde{W}_{3,2}^{1}\left(y^{\prime}, t\right)}{\partial y_{2}}-\frac{\partial \widetilde{W}_{2,2}^{1}\left(y^{\prime}, t\right)}{\partial y_{3}}\right)+s^{2}(t) \\
& -\frac{\partial \widetilde{V}_{2}^{0}\left(y^{\prime}, t\right)}{\partial t}
\end{aligned}
$$




$$
\begin{aligned}
& -\alpha \Delta_{y^{\prime}} \widetilde{W}_{2,2}^{2}\left(y^{\prime}, t\right) \\
& -\beta \frac{\partial}{\partial y_{2}}\left(\frac{\partial \widetilde{W}_{2,2}^{2}\left(y^{\prime}, t\right)}{\partial y_{2}}+\frac{\partial \widetilde{W}_{3,2}^{2}\left(y^{\prime}, t\right)}{\partial y_{3}}\right) \\
& =a \frac{\partial \widetilde{V}_{2}^{1}\left(y^{\prime}, t\right)}{\partial y_{3}}-\frac{\partial \widetilde{W}_{2,2}^{0}\left(y^{\prime}, t\right)}{\partial t}-2 a \widetilde{W}_{2,2}^{0}\left(y^{\prime}, t\right), \\
& -\alpha \Delta_{y^{\prime}} \widetilde{W}_{3,2}^{2}\left(y^{\prime}, t\right) \\
& -\beta \frac{\partial}{\partial y_{3}}\left(\frac{\partial \widetilde{W}_{2,2}^{2}\left(y^{\prime}, t\right)}{\partial y_{2}}+\frac{\partial \widetilde{W}_{3,2}^{2}\left(y^{\prime}, t\right)}{\partial y_{3}}\right) \\
& =-a \frac{\partial \widetilde{V}_{2}^{1}\left(y^{\prime}, t\right)}{\partial y_{2}}-\frac{\partial \widetilde{W}_{3,2}^{0}\left(y^{\prime}, t\right)}{\partial t}-2 a \widetilde{W}_{3,2}^{0}\left(y^{\prime}, t\right),
\end{aligned}
$$$$
\left.\widetilde{V}_{2}^{2}\left(y^{\prime}, t\right)\right|_{\partial B}=0,
$$$$
\left.\widetilde{W}_{2,2}^{2}\left(y^{\prime}, t\right)\right|_{\partial B}=0,
$$$$
\left.\widetilde{W}_{3,2}^{2}\left(y^{\prime}, t\right)\right|_{\partial B}=0,
$$$$
\int_{\partial B} \widetilde{V}_{2}^{2}\left(y^{\prime}, t\right) d y^{\prime}=H^{2}(t),
$$

where $H^{2}(t)$ is given by

$$
\begin{aligned}
& H^{2}(t)=F^{2}(t)-\int_{B} \widetilde{V}_{1}^{2}\left(y^{\prime}, t\right) d y^{\prime}=F^{2}(t) \\
& -\frac{\pi R^{6} \alpha(d / d t) \widetilde{f}^{0}(t)-\pi R^{6} a^{2} \widetilde{f}^{0}(t)}{96 \mu^{2} \alpha} \\
& -\frac{8 \pi \mu \alpha R^{4} \widetilde{f}^{2}(t)+\pi R^{6}\left(a^{2} \widetilde{f}^{0}(t)-2 \alpha(d / d t) \tilde{f}^{0}(t)\right)}{264 \mu^{3} \alpha} .
\end{aligned}
$$

Employing the expressions for the zero-order approximation given by (56) and (59) and for the first-order corrector given by (63) and (66), from (67) we get the following:

$$
\begin{aligned}
-\mu \Delta_{y^{\prime}} \widetilde{V}_{2}^{2}\left(y^{\prime}, t\right) \\
=\left(-\frac{a^{2} H^{0}(t)}{4 \mu \alpha \kappa_{0}}+\frac{(d / d t) H^{0}(t)}{4 \mu \kappa_{0}}\right)\left(y_{2}^{2}+y_{3}^{2}\right)
\end{aligned}
$$

$$
\begin{aligned}
& +s^{2}(t)+\frac{R^{2} a^{2} H^{0}(t)-2 \alpha R^{2} H^{0}(t)}{8 \mu \alpha \kappa_{0}}, \\
& -\alpha \Delta_{y^{\prime}} \widetilde{W}_{2,2}^{2}\left(y^{\prime}, t\right) \\
& -\beta \frac{\partial}{\partial y_{2}}\left(\frac{\partial \widetilde{W}_{2,2}^{2}\left(y^{\prime}, t\right)}{\partial y_{2}}+\frac{\partial \widetilde{W}_{3,2}^{2}\left(y^{\prime}, t\right)}{\partial y_{3}}\right) \\
& =\frac{-a H^{1}(t)}{2 \mu \kappa_{1}} y_{3}, \\
& -\alpha \Delta_{y^{\prime}} \widetilde{W}_{3,2}^{2}\left(y^{\prime}, t\right) \\
& -\beta \frac{\partial}{\partial y_{3}}\left(\frac{\partial \widetilde{W}_{2,2}^{2}\left(y^{\prime}, t\right)}{\partial y_{2}}+\frac{\partial \widetilde{W}_{3,2}^{2}\left(y^{\prime}, t\right)}{\partial y_{3}}\right) \\
& =\frac{a H^{1}(t)}{2 \mu \kappa_{1}} y_{2}, \\
& \left.\widetilde{V}_{2}^{2}\left(y^{\prime}, t\right)\right|_{\partial B}=0, \\
& \left.\widetilde{W}_{2,2}^{2}\left(y^{\prime}, t\right)\right|_{\partial B}=0, \\
& \left.\widetilde{W}_{3,2}^{2}\left(y^{\prime}, t\right)\right|_{\partial B}=0,
\end{aligned}
$$$$
\int_{B} \widetilde{V}_{2}^{2}\left(y^{\prime}, t\right) d y^{\prime}=H^{2}(t) .
$$

We can now explicitly compute the velocity corrector $\widetilde{V}_{2}^{2}\left(y^{\prime}, t\right)$ as

$$
\begin{aligned}
& \widetilde{V}_{2}^{2}\left(y^{\prime}, t\right)=-\frac{1}{16 \mu}\left(-\frac{a^{2} H^{0}(t)}{4 \mu \alpha \kappa_{0}}+\frac{(d / d t) H^{0}(t)}{4 \mu \kappa_{0}}\right) \\
& \cdot\left(\left|y^{\prime}\right|^{4}-R^{4}\right) \\
& -\frac{1}{4 \mu}\left(s^{2}(t)+\frac{R^{2} a^{2} H^{0}(t)-2 \alpha R^{2} H^{0}(t)}{8 \mu \alpha \kappa_{0}}\right) \\
& \cdot\left(\left|y^{\prime}\right|^{2}-R^{2}\right),
\end{aligned}
$$

where we choose $s^{2}(t)$ to satisfy the flux condition:

$s^{2}(t)$

$=\frac{H^{2}(t)+(1 / 16 \mu)\left(-a^{2} H^{0}(t) / 4 \mu \alpha \kappa_{0}+(d / d t) H^{0}(t) / 4 \mu \kappa_{0}\right)\left(-2 \pi R^{6} / 3\right)+(1 / 4 \mu)\left(\left(R^{2} a^{2} H^{0}(t)-2 \alpha R^{2} H^{0}(t)\right) / 8 \mu \alpha \kappa_{0}\right)\left(-R^{4} \pi / 2\right)}{-(1 / 4 \mu)\left(-R^{4} \pi / 2\right)}$. 
Similarly, we can explicitly compute the corrector for the microrotation:

$$
\begin{aligned}
& \widetilde{W}_{2,2}^{2}\left(y^{\prime}, t\right)=\left(\left|y^{\prime}\right|^{2}-R^{2}\right) \frac{a H^{1}(t)}{16 \mu \alpha \kappa_{1}} y_{3}, \\
& \widetilde{W}_{3,2}^{2}\left(y^{\prime}, t\right)=\left(\left|y^{\prime}\right|^{2}-R^{2}\right) \frac{-a H^{1}(t)}{16 \mu \alpha \kappa_{1}} y_{2} .
\end{aligned}
$$

\section{Asymptotic Approximation}

The asymptotic approximation related to the micropolar heat problem (11) has the following form:

$$
\begin{aligned}
\widetilde{V}_{1}^{\mathrm{apr}}\left(x^{\prime}, t\right)= & \widetilde{V}_{1}^{0}\left(\frac{x^{\prime}}{\epsilon}, t\right)+\epsilon \widetilde{V}_{1}^{1}\left(\frac{x^{\prime}}{\epsilon}, t\right) \\
& +\epsilon^{2} \widetilde{V}_{1}^{2}\left(\frac{x^{\prime}}{\epsilon}, t\right), \\
\widetilde{W}_{2,1}^{\mathrm{apr}}\left(x^{\prime}, t\right)= & \widetilde{W}_{2,1}^{0}\left(\frac{x^{\prime}}{\epsilon}, t\right)+\epsilon \widetilde{W}_{2,1}^{1}\left(\frac{x^{\prime}}{\epsilon}, t\right) \\
& +\epsilon^{2} \widetilde{W}_{2,1}^{2}\left(\frac{x^{\prime}}{\epsilon}, t\right), \\
\widetilde{W}_{3,1}^{\mathrm{apr}}\left(x^{\prime}, t\right)= & \widetilde{W}_{3,1}^{0}\left(\frac{x^{\prime}}{\epsilon}, t\right)+\epsilon \widetilde{W}_{3,1}^{1}\left(\frac{x^{\prime}}{\epsilon}, t\right) \\
& +\epsilon^{2} \widetilde{W}_{3,1}^{2}\left(\frac{x^{\prime}}{\epsilon}, t\right) .
\end{aligned}
$$

Note we have calculated all the terms explicitly; that is, $\left(\widetilde{V}_{1}^{j}\left(x^{\prime} / \epsilon, t\right), \widetilde{W}_{2,1}^{j}\left(x^{\prime} / \epsilon, t\right), \widetilde{W}_{3,1}^{j}\left(x^{\prime} / \epsilon, t\right)\right), j=0,1,2$, are given by (27) and (28a)-(28b), (34), (36), (43), and (46)-(47).

For the micropolar inverse problem (12), we obtain the following:

$$
\begin{aligned}
V_{2}^{\mathrm{apr}}\left(x^{\prime}, t\right)= & \widetilde{V}_{2}^{0}\left(\frac{x^{\prime}}{\epsilon}, t\right)+\epsilon \widetilde{V}_{2}^{1}\left(\frac{x^{\prime}}{\epsilon}, t\right) \\
& +\epsilon^{2} \widetilde{V}_{2}^{2}\left(\frac{x^{\prime}}{\epsilon}, t\right), \\
W_{2,2}^{\mathrm{apr}}\left(x^{\prime}, t\right)= & \widetilde{W}_{2,2}^{0}\left(\frac{x^{\prime}}{\epsilon}, t\right)+\epsilon \widetilde{W}_{2,2}^{1}\left(\frac{x^{\prime}}{\epsilon}, t\right) \\
& +\epsilon^{2} \widetilde{W}_{2,2}^{2}\left(\frac{x^{\prime}}{\epsilon}, t\right), \\
W_{3,2}^{\mathrm{apr}}\left(x^{\prime}, t\right)= & \widetilde{W}_{3,2}^{0}\left(\frac{x^{\prime}}{\epsilon}, t\right)+\epsilon \widetilde{W}_{3,2}^{1}\left(\frac{x^{\prime}}{\epsilon}, t\right) \\
& +\epsilon^{2} \widetilde{W}_{3,2}^{2}\left(\frac{x^{\prime}}{\epsilon}, t\right), \\
s^{\mathrm{apr}}(t)= & s^{0}(t)+\epsilon s^{1}(t)+\epsilon^{2} s^{2}(t),
\end{aligned}
$$

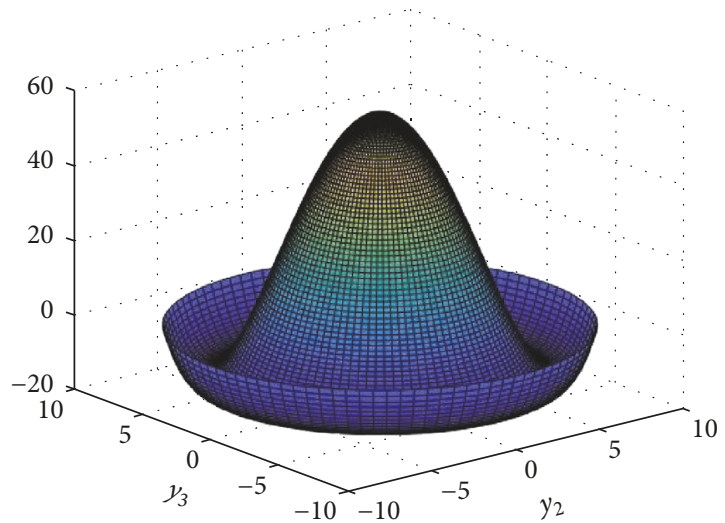

FIGURE 2: Second-order velocity corrector $\widetilde{V}_{2}^{2}$.

where $\left(\widetilde{V}_{2}^{j}\left(x^{\prime} / \epsilon, t\right), \widetilde{W}_{2,2}^{j}\left(x^{\prime} / \epsilon, t\right), \widetilde{W}_{3,2}^{j}\left(x^{\prime} / \epsilon, t\right), s^{j}(t)\right), j=$ $0,1,2$, are given by the explicit formulae (56)-(59), (63)-(66), and (70)-(72).

Collecting the above results, we arrive at the asymptotic approximation $\left(v^{\mathrm{apr}}\left(x^{\prime}, t\right), w_{2}^{\mathrm{apr}}\left(x^{\prime}, t\right), w_{3}^{\mathrm{apr}}\left(x^{\prime}, t\right), q^{\mathrm{apr}}(t)\right)$ for problem (7)-(9):

$$
\begin{aligned}
v^{\mathrm{apr}}\left(x^{\prime}, t\right) & =V_{1}^{\mathrm{apr}}\left(x^{\prime}, t\right)+V_{2}^{\mathrm{apr}}\left(x^{\prime}, t\right), \\
w_{2}^{\mathrm{apr}}\left(x^{\prime}, t\right) & =\widetilde{W}_{2,1}^{\mathrm{apr}}\left(x^{\prime}, t\right)+\widetilde{W}_{2,2}^{\mathrm{apr}}\left(x^{\prime}, t\right), \\
w_{3}^{\mathrm{apr}}\left(x^{\prime}, t\right) & =\widetilde{W}_{3,1}^{\mathrm{apr}}\left(x^{\prime}, t\right)+\widetilde{W}_{3,2}^{\mathrm{apr}}\left(x^{\prime}, t\right), \\
q^{\mathrm{apr}}(t) & =\frac{1}{\epsilon^{2}} s^{\mathrm{apr}}(t) .
\end{aligned}
$$

It should be noted that the above asymptotic approximation was computed to satisfy (7), the boundary conditions (8a) $-(8 c)$, and the flux condition (9). The initial conditions (8d)-(8f) were not taken into account in the process due to the fact that the time derivative appears only in the system for the second-order corrector (namely, as the time derivative of the zero-order approximation for the velocity and microrotation which is a known function on the righthand side). Thus, taking into account the initial conditions while computing the correctors would yield overdetermined systems for the unknown terms in the asymptotic expansion. This essentially means that a boundary-layer-in-time phenomena appears; that is, near $t=0$, we have some influence of the initial conditions that cannot be captured by the regular expansion. We can fix that by introducing the appropriate boundary-layer correctors, as proposed in [10] for Newtonian flow. However, it can be proved that those correctors would have the exponential decay towards zero; that is, it would not affect the approximation outside the boundary layers in time. More precisely, it would only serve for the convergence proof, namely, to derive satisfactory error estimates in the appropriate norms. Detailed analysis of the boundary layers together with the rigorous justification of the complete asymptotic expansion (up to a general order $J$ ) will be brought in the forthcoming paper by the authors of the present paper. 


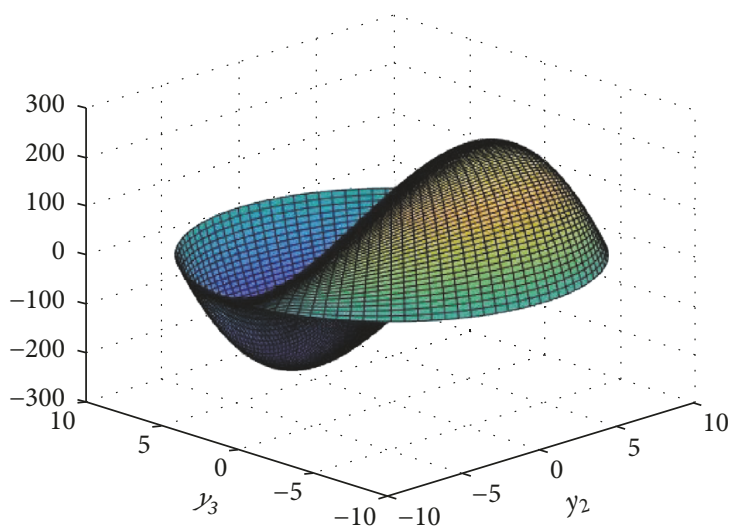

(a)

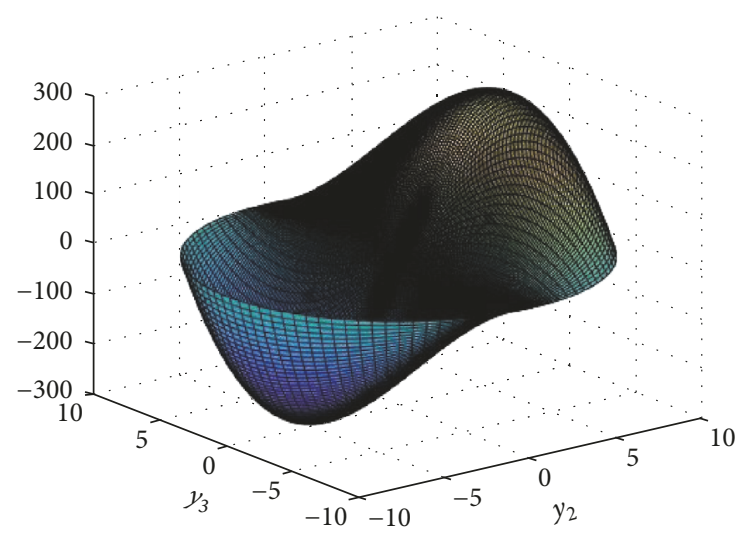

(b)

FIGURE 3: Second-order microrotation corrector $\widetilde{W}_{2,2}^{2}$ (a) and $\widetilde{W}_{3,2}^{2}$ (b).

\section{Numerical Example}

Finally, in this section, we aim to visually present the derived asymptotic solution for problem (7)-(9). We take the following values for the viscosity constants (see e.g., [21, 22]):

$$
\begin{aligned}
& \nu=2.9 \times 10^{-3}, \\
& v_{r}=2.32 \times 10^{-4}, \\
& a=2 v_{r}=4.64 \times 10^{-4}, \\
& \mu=\nu+v_{r}, \\
& \alpha=c_{a}+c_{d}=10^{-6}, \\
& \beta=c_{0}+c_{d}-c_{a}=10^{-6} .
\end{aligned}
$$

The domain is circular with radius $R=10$. Furthermore, all the visualizations are done for fixed time $t=10$ and given flow rates $F^{0}(t)=t+50, F^{1}(t)=t^{2}$, and $F^{2}(t)=t^{3}$. The linear and angular momentum force functions are neglected; namely, $\widetilde{f}_{1}(t) \equiv \widetilde{g}_{2}(t) \equiv \widetilde{g}_{3}(t) \equiv 0$. Therefore, it follows that $\left(V_{1}^{\epsilon,[2]}\left(x^{\prime}, t\right), W_{2,1}^{\epsilon,[2]}\left(x^{\prime}, t\right), W_{3,1}^{\epsilon,[2]}\left(x^{\prime}, t\right)\right) \equiv 0$ (see (27), (28a)(28b), (34), (36), (43), and (46)-(47)) and $F^{j}(t) \equiv H^{j}(t), j=$ $0,1,2$ (see (55), (61), and (68)). As a result, the asymptotic solution reads

$$
\begin{aligned}
v^{\mathrm{apr}}\left(x^{\prime}, t\right)= & \widetilde{V}_{2}^{0}\left(\frac{x^{\prime}}{\epsilon}, t\right)+\epsilon \widetilde{V}_{2}^{1}\left(\frac{x^{\prime}}{\epsilon}, t\right) \\
& +\epsilon^{2} \widetilde{V}_{2}^{2}\left(\frac{x^{\prime}}{\epsilon}, t\right), \\
w_{2}^{\mathrm{apr}}\left(x^{\prime}, t\right)= & \widetilde{W}_{2,2}^{0}\left(\frac{x^{\prime}}{\epsilon}, t\right)+\epsilon \widetilde{W}_{2,2}^{1}\left(\frac{x^{\prime}}{\epsilon}, t\right) \\
& +\epsilon^{2} \widetilde{W}_{2,2}^{2}\left(\frac{x^{\prime}}{\epsilon}, t\right),
\end{aligned}
$$

$$
\begin{aligned}
w_{3}^{\mathrm{apr}}\left(x^{\prime}, t\right)= & \widetilde{W}_{3,2}^{0}\left(\frac{x^{\prime}}{\epsilon}, t\right)+\epsilon \widetilde{W}_{3,2}^{1}\left(\frac{x^{\prime}}{\epsilon}, t\right) \\
& +\epsilon^{2} \widetilde{W}_{3,2}^{2}\left(\frac{x^{\prime}}{\epsilon}, t\right),
\end{aligned}
$$

where $\left(\widetilde{V}_{2}^{j}\left(x^{\prime} / \epsilon, t\right), \widetilde{W}_{2,2}^{j}\left(x^{\prime} / \epsilon, t\right), \widetilde{W}_{3,2}^{j}\left(x^{\prime} / \epsilon, t\right)\right), j=0,1,2$, have been explicitly computed in (56)-(59), (63)-(66), and (70)-(72).

In the following, we depict the second-order correctors for the velocity and microrotation (see Figures 2 and 3) as well as the asymptotic approximation for the velocity and microrotation, given by (77), for $\epsilon=0.1$ (see Figures 4 and 5). We omit the visualizations for the zero-order approximations and first-order correctors since they are of the classical Poiseuille form (for the velocity) and of the same form as the second-order corrector for the microrotation.

6.1. Second-Order Correctors. We notice that the velocity second-order corrector is of shape and scale that will affect our asymptotic approximation if $\epsilon$ is not too small (e.g., $\epsilon=0.1)$. This has been visually verified in Figure 4 . The microrotation second-order corrector does not affect the asymptotic approximation in a significant way as the firstorder corrector is of the same shape and similar scale. Note that the second-order correctors are scaled with the small parameter $\epsilon^{2}$ in the asymptotic approximation (77).

6.2. Asymptotic Approximation. In Figure 4 we compare our asymptotic approximation $v^{\text {apr }}$ and the zero-order approximation $\widetilde{V}_{2}^{0}$. The second-order corrector for the velocity affects the approximation in the whole domain, with a clear impact near the boundary of the domain, correcting the Poiseuille zero-order approximation, and we can clearly observe the effect visually. The microrotation approximation is the scaled sum of the first- and second-order corrector, thus having the same form as the first- and second-order correctors. 


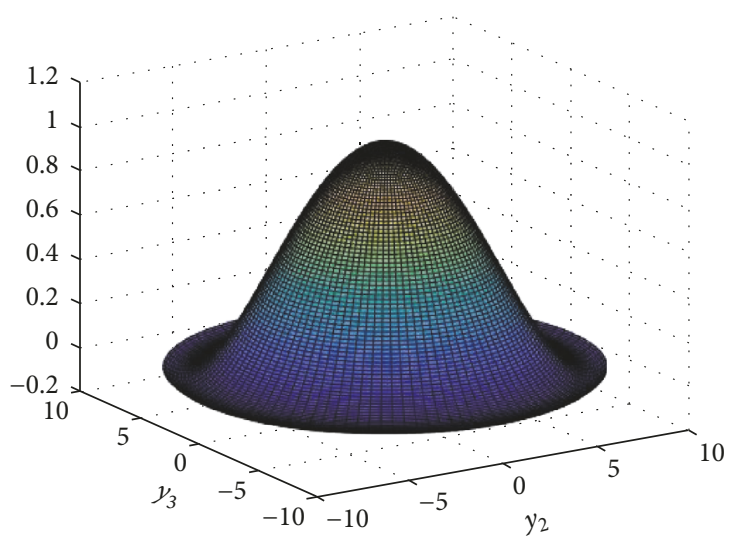

(a)

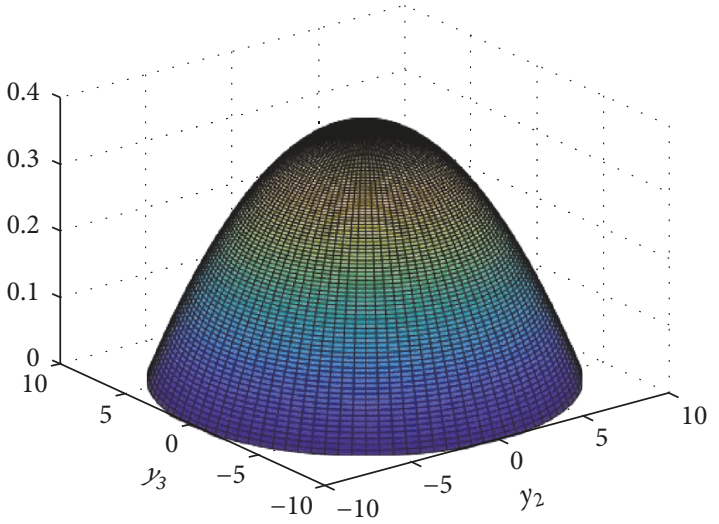

(b)

FIGURE 4: Velocity approximation $v^{\text {apr }}$ (a) and zero-order approximation $\widetilde{V}_{2}^{0}$ (b).

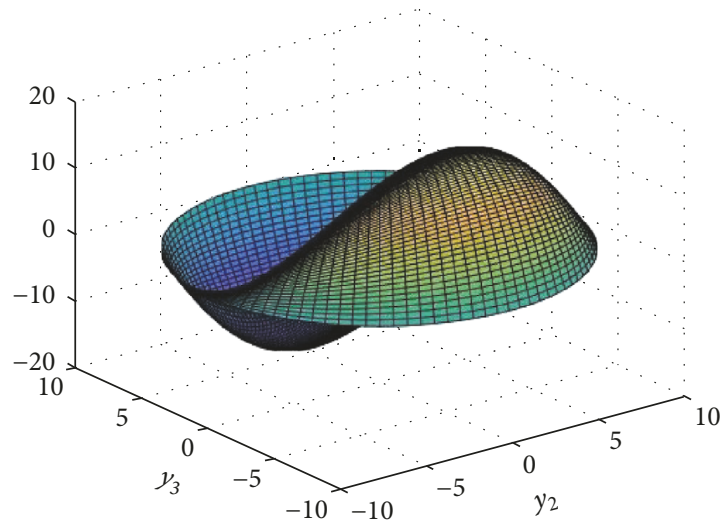

(a)

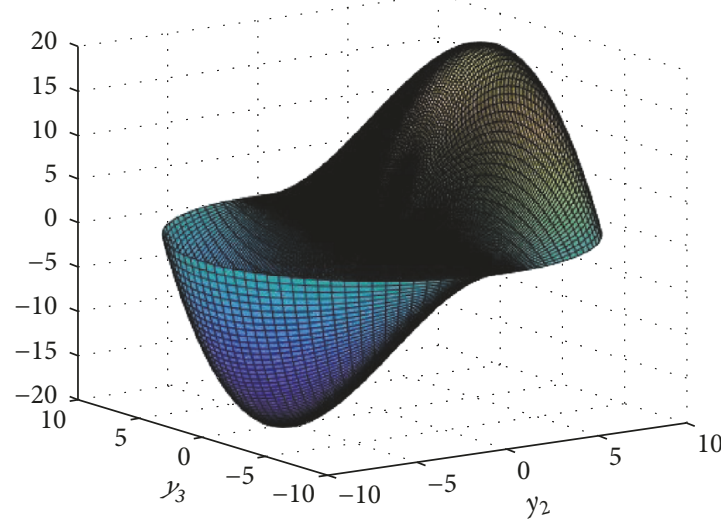

(b)

FIGURE 5: Microrotation approximation $w_{2}^{\text {apr }}$ (a) and $w_{3}^{\text {apr }}$ (b).

\section{Conflicts of Interest}

The authors declare that they have no conflicts of interest.

\section{Acknowledgments}

The authors of this work have been supported by the Croatian Science Foundation (Scientific Project 3955: Mathematical Modeling and Numerical Simulations of Processes in Thin or Porous Domains).

\section{References}

[1] E. L. Aero, A. N. Bulygin, and E. V. Kuvshinskii, "Asymmetric hydromechanics," Journal of Applied Mathematics and Mechanics, vol. 29, no. 2, pp. 333-346, 1965.

[2] A. C. Eringen, “Theory of micropolar fluids," Journal of Applied Mathematics and Mechanics, vol. 16, pp. 1-18, 1966.

[3] G. Lukaszewicz, Micropolar Fluids: Theory and Application, Birkhäuser, Boston, Mass, USA, 1999.

[4] C.-L. Chang, "Numerical simulation of micropolar fluid flow along a flat plate with wall conduction and buoyancy effects,"
Journal of Physics D: Applied Physics, vol. 39, no. 6, pp. 1132-1140, 2006.

[5] A. Ishak, Y. Y. Lok, and I. Pop, "Stagnation point flow over a shrinking sheet in micropolar fluid," Chemical Engineering Communications, vol. 197, no. 11, pp. 1417-1427, 2010.

[6] I. Abdullah and N. Amin, "A micropolar fluid model of blood flow through a tapered artery with a stenosis," Mathematical Methods in the Applied Sciences, vol. 33, no. 16, pp. 1910-1923, 2010.

[7] D. Dupuy, G. P. Panasenko, and R. Stavre, "Asymptotic methods for micropolar fluids in a tube structure," Mathematical Models and Methods in Applied Sciences, vol. 14, no. 5, pp. 735-758, 2004.

[8] I. Pažanin, "On the micropolar flow in a circular pipe: the effects of the viscosity coefficients," Theoretical \& Applied Mechanics Letters, vol. 1, Article ID 062004, 2011.

[9] E. Marušić-Paloka, I. Pažanin, and M. Radulović, "Flow of a micropolar fluid through a channel with small boundary perturbation," Zeitschrift fur Naturforschung A, vol. 71, no. 7, pp. 607-619, 2016.

[10] G. Panasenko and K. Pileckas, "Asymptotic analysis of the nonsteady viscous flow with a given flow rate in a thin pipe," 
Applicable Analysis: An International Journal, vol. 91, no. 3, pp. 559-574, 2012.

[11] G. Panasenko and K. Pileckas, "Asymptotic analysis of the nonsteady Navier-Stokes equations in a tube structure. I. The case without boundary-layer-in-time," Nonlinear Analysis: Theory, Methods \& Applications, vol. 122, pp. 125-168, 2015.

[12] G. Panasenko and K. Pileckas, "Asymptotic analysis of the nonsteady Navier-Stokes equations in a tube structure. II. General case," Nonlinear Analysis: Theory, Methods \& Applications, vol. 125, pp. 582-607, 2015.

[13] I. Pažanin, "Effective flow of micropolar fluid through a thin or long pipe," Mathematical Problems in Engineering, vol. 2011, Article ID 127070, 18 pages, 2011.

[14] I. Pažanin, "Asymptotic behavior of micropolar fluid flow through a curved pipe," Acta Applicandae Mathematicae, vol. 116, no. 1, pp. 1-25, 2011.

[15] I. Pažanin, "Investigation of micropolar fluid flow in a helical pipe via asymptotic analysis," Communications in Nonlinear Science and Numerical Simulation, vol. 18, no. 3, pp. 528-540, 2013.

[16] M. Beneš and I. Pažanin, "Effective flow of incompressible micropolar fluid through a system of thin pipes," Acta Applicandae Mathematicae, vol. 143, pp. 29-43, 2016.

[17] A. A. El-Bary, "Exponential solution of a problem of twodimensional motion of micropolar fluid in a half-plane," Applied Mathematics and Computation, vol. 165, no. 1, pp. 81-93, 2005.

[18] N. A. Khan and M. Jamil, "Analytic solution for creping flow of an unsteady micropolar fluid," International Journal of Applied Mathematics and Mechanics, vol. 5, pp. 39-47, 2009.

[19] A. Zaman, N. Ali, and O. Anwar Bég, "Numerical simulation of unsteady micropolar hemodynamics in a tapered catheterized artery with a combination of stenosis and aneurysm," Medical \& Biological Engineering \& Computing, vol. 54, no. 9, pp. 14231436, 2016.

[20] S. H. M. Saleh, N. M. Arifin, R. Nazar, and I. Pop, "Unsteady micropolar fluid over a permeable curved stretching shrinking surface," Mathematical Problems in Engineering, vol. 2017, Article ID 3085249, 13 pages, 2017.

[21] I. Papautsky, J. Brazzle, T. Ameel, and A. B. Frazier, "Laminar fluid behavior in microchannels using micropolar fluid theory," Sensors and Actuators A: Physical, vol. 73, no. 1-2, pp. 101-108, 1999.

[22] K. P. Madasu and M. K. Gurdatta, "Steady rotation of micropolar fluid sphere in concentric spherical container," Procedia Engineering, vol. 127, pp. 469-475, 2015. 


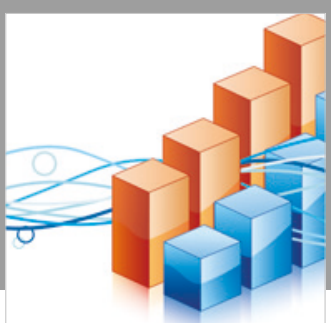

Advances in

Operations Research

\section{-n-m}
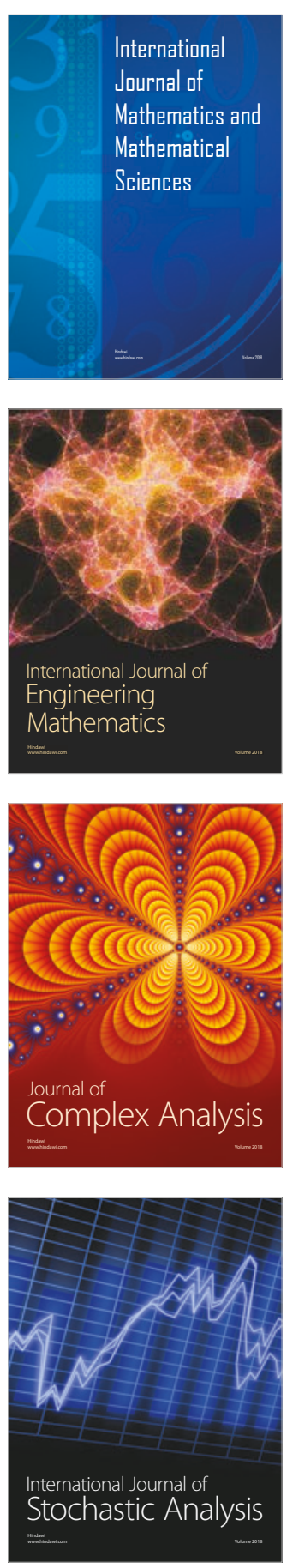
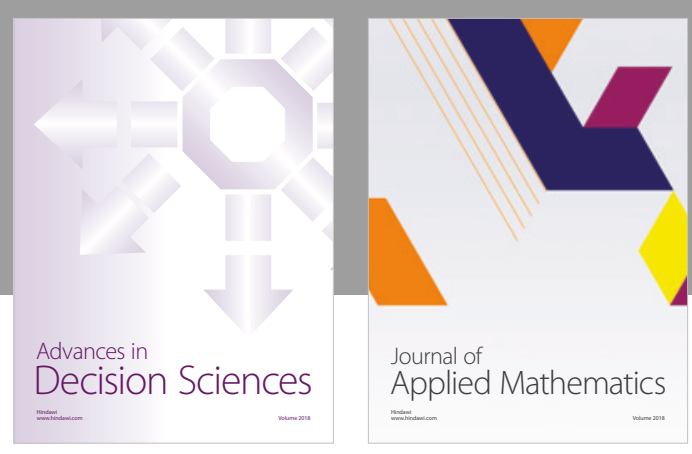

Journal of

Applied Mathematics
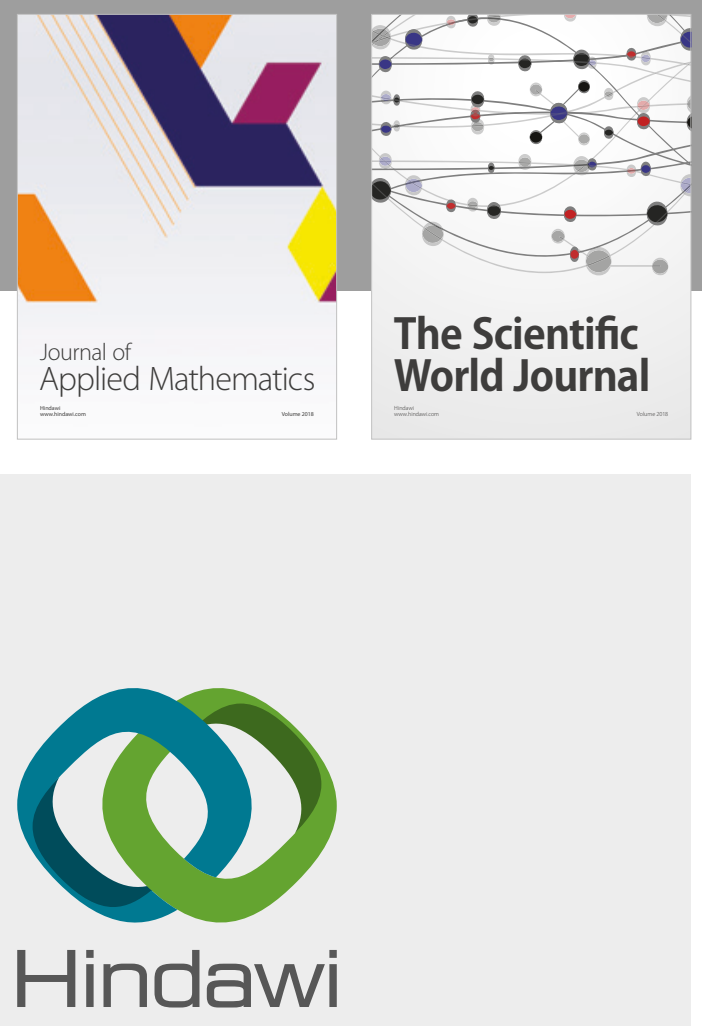

Submit your manuscripts at

www.hindawi.com

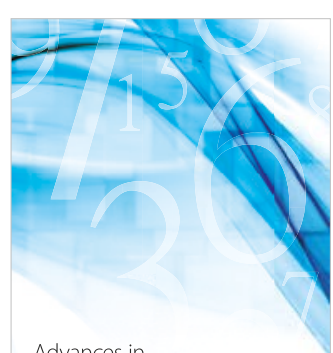

Advances in
Numerical Analysis
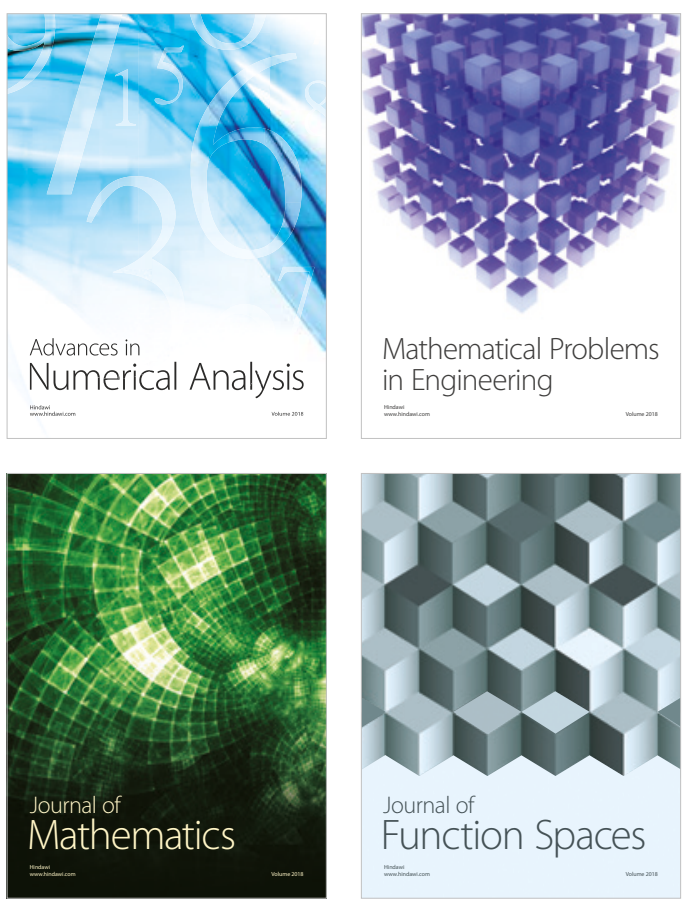

Mathematical Problems in Engineering

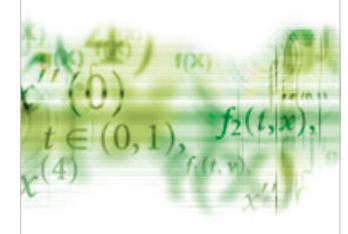

International Journal of

Differential Equations

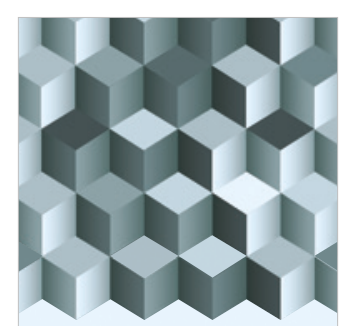

Journal of

Function Spaces

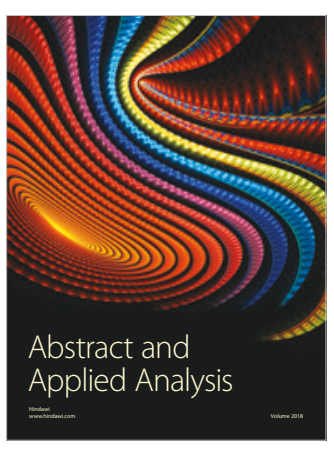

The Scientific

World Journal

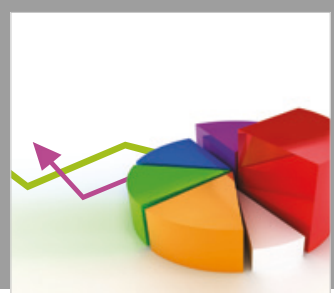

Journal of

Probability and Statistics
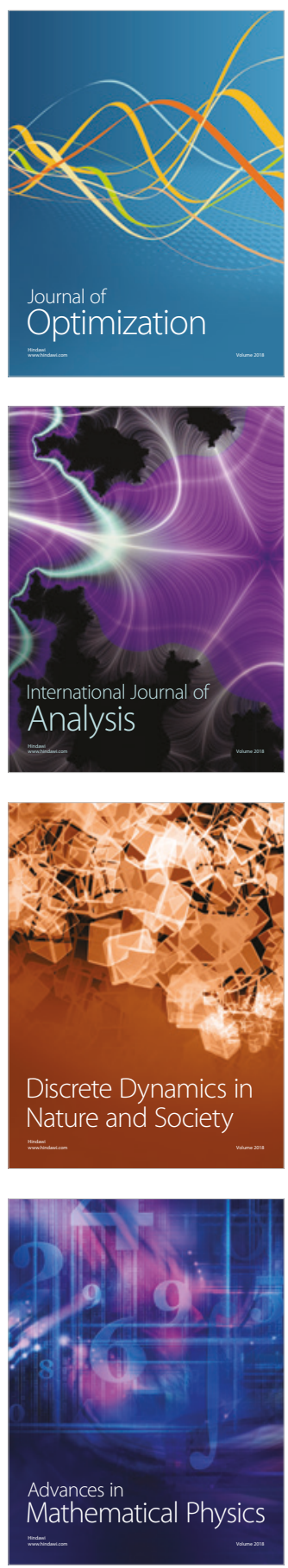